\title{
Quotient-space boundary element methods for scattering at complex screens
}

\author{
Xavier Claeys ${ }^{1} \cdot$ Lorenzo Giacomel $^{2} \cdot$ Ralf Hiptmair $^{3}$ (1) \\ Carolina Urzúa-Torres ${ }^{4}$
}

Received: 24 June 2020 / Accepted: 5 March 2021 / Published online: 29 March 2021

(C) The Author(s) 2021

\begin{abstract}
A complex screen is an arrangement of panels that may not be even locally orientable because of junction lines. A comprehensive trace space framework for first-kind variational boundary integral equations on complex screens has been established in Claeys and Hiptmair (Integr Equ Oper Theory 77:167-197, 2013. https://doi.org/10.1007/ s00020-013-2085-x) for the Helmholtz equation, and in Claeys and Hiptmair (Integr Equ Oper Theory 84:33-68, 2016. https://doi.org/10.1007/s00020-015-2242-5) for Maxwell's equations in frequency domain. The gist is a quotient space perspective that allows to make sense of jumps of traces as factor spaces of multi-trace spaces modulo single-trace spaces without relying on orientation. This paves the way for formulating first-kind boundary integral equations in weak form posed on energy trace spaces. In this article we extend that idea to the Galerkin boundary element (BE) discretization of first-kind boundary integral equations. Instead of trying to approximate
\end{abstract}

Communicated by Ragnar Winther.

The work of C. Urzúa-Torres was partly supported by ETHIRA Grant ETH-04 13-2.

$凶 \quad$ Ralf Hiptmair

hiptmair@sam.math.ethz.ch

Xavier Claeys

xavier.claeys@upmc.fr

Lorenzo Giacomel

glorenzo@ethz.ch

Carolina Urzúa-Torres

C.A.UrzuaTorres@tudelft.nl

1 Laboratoire Jacques-Louis Lions, Sorbonne Université, Univ Paris-Diderot SPC, CNRS, INRIA, équipe ALPINES, Pairs, France

2 Beams Department, CERN, Geneva, Switzerland

3 Seminar for Applied Mathematics, ETH Zurich, Zurich, Switzerland

4 Delft Institute of Applied Mathematics, TU Delft, Delft, The Netherlands 
jumps directly, the new quotient space boundary element method employs a Galerkin BE approach in multi-trace boundary element spaces. This spawns discrete boundary integral equations with large null spaces comprised of single-trace functions. Yet, since the right-hand-sides of the linear systems of equations are consistent, Krylov subspace iterative solvers like GMRES are not affected by the presence of a kernel and still converge to a solution. This is strikingly confirmed by numerical tests.

Keywords Complex screens · Galerkin boundary element method · Quotient space boundary element method

Mathematics Subject Classification 65N38 - 78M15

\section{Scattering at multi-screens}

We are concerned with the scattering of acoustic or electromagnetic waves at objects like those displayed in Fig. 1, i.e. geometries composed of essentially twodimensional piecewise smooth surfaces joined together. These objects can be regarded as non-penetrable, more precisely: sound-soft, sound-hard, or perfectly conducting, respectively. This implies vanishing traces of some fields on their "surface". We face boundary value problems posed on the unbounded complement of the scattering object. Our goal is to solve them, that is, to compute the scattered wave by means of a Galerkin boundary element method (BEM).

To that end, we recast the boundary value problems as variational boundary integral equations (BIEs) posed in spaces of functions on the surface of the scattering object. For simple screens this is well established [31, Section 3.5.3]. Here, we call a simple screen an orientable, piecewise smooth two-dimensional bounded manifold $\Gamma$ embedded in $3 \mathrm{D}$ space $\mathbb{R}^{3}$. In this case, coercive variational first-kind boundary integral equations arise, known as weakly singular and hypersingular BIEs in the acoustic setting [14, 15,33], and as Electric Field Integral Equation (EFIE) for electromagnetics [4]. These BIEs are set in Sobolev spaces of jumps of suitable field traces, in $\widetilde{H}^{-\frac{1}{2}}(\Gamma)$ and $\widetilde{H}^{\frac{1}{2}}(\Gamma)$, respectively, for acoustics [24, Ch. 3], and in $\widetilde{\mathbf{H}}^{-\frac{1}{2}}\left(\operatorname{curl}_{\Gamma}, \Gamma\right)$ for the EFIE. For these
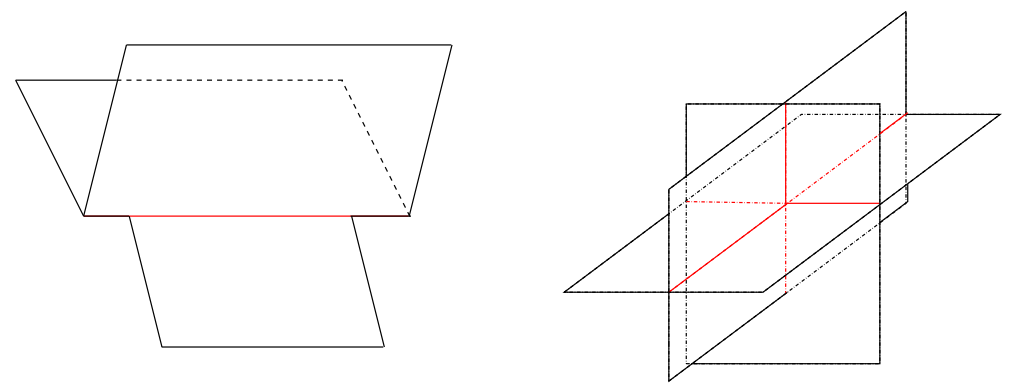

Fig. 1 Two examples of multi-screen geometries; junctions lines colored red 
trace spaces, conforming boundary element subspaces are readily available, and they give rise to Galerkin approximations whose numerical analysis is fairly mature [5].

Obviously, for two-dimensional objects $\Gamma$, like those shown in Fig. 1, which are not globally orientable, the notion of jumps becomes problematic. It is not straightforward how to adapt the jump trace spaces from the simple-screen setting to more general situations. A breakthrough was achieved in [8] for the BIEs of acoustic scattering, and in [9] towards generalizing the EFIE. The main idea was to consistently view trace spaces, including those for jumps, from the perspective of quotient spaces and to start from multi-valued traces. We survey these results in Sect. 2.

An important step in [8,9] was the rigorous characterization of geometries as those of Fig. 1. The authors introduced the class of multi-screens and defined them as follows, see [8, Section 2] for more details:

Definition 1.1 (Lipschitz Partition [8, Definition 2.2]) A Lipschitz partition of $\mathbb{R}^{d}$, $d=2,3$, is a finite collection of Lipschitz open sets $\left(\Omega_{j}\right)_{j=0 \ldots n}$ such that $\mathbb{R}^{d}=$ $\cup_{j=0}^{n} \bar{\Omega}_{j}$ and $\Omega_{j} \cap \Omega_{k}=\emptyset$, if $j \neq k$.

Definition 1.2 (Multi-screen [8, Definition 2.3]) A multi-screen is a subset $\Gamma \subset \mathbb{R}^{d}$ such that there exists a Lipschitz partition $\mathbb{R}^{d}$ denoted $\left(\Omega_{j}\right)_{j=0 \ldots n}$ satisfying $\Gamma \subset$ $\cup_{j=0}^{n} \partial \Omega_{j}$ and such that for each $j=0 \ldots n$, we have $\bar{\Gamma} \cap \partial \Omega_{j}=\bar{\Gamma}_{j}$ where $\bar{\Gamma}_{j} \subset \partial \Omega_{j}$ is some Lipschitz screen in the sense of Buffa-Christiansen [4, section 1.1].

We want to take the cue from the theoretical investigations to develop Galerkin BEM for multi-screens in 3D $(d=3)$. Of course, application of the BEM entails restricting the set of admissible multi-screens. We confine ourselves to those that are the union of (closed) triangles such that the intersection of two triangles is either empty, a single point, or a common edge of both. Fittingly, we call these shapes triangulated multi-screens, and some of them are rendered in Fig. 2.

We are going to present an approach that will yield a Galerkin BEM discretization of the boundary integral equations for acoustic and electromagnetic scattering at general triangulated multi-screens. We rely on minimal information about the geometry and
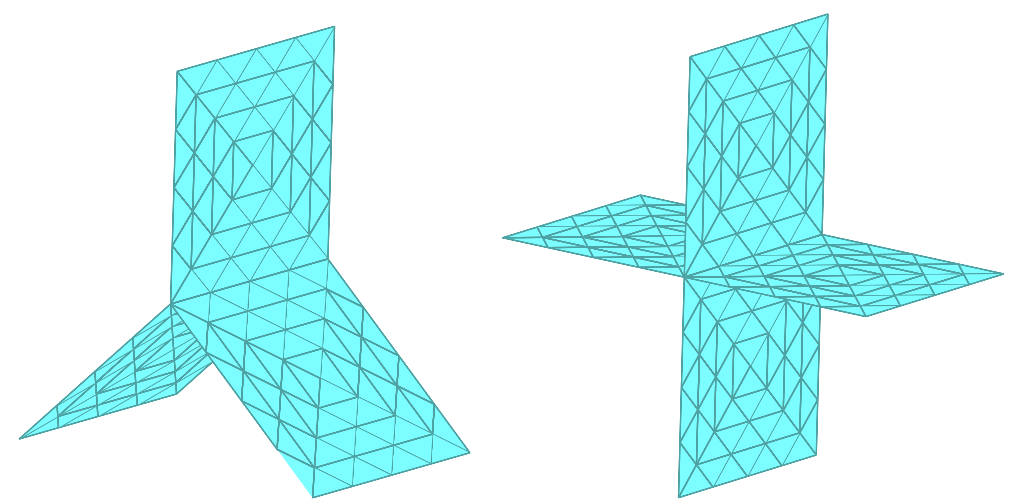

Fig. 2 Two examples of triangulated multi-screens: triple- and quadruple junctions 
no special treatment of "junction lines" or "joints" is necessary. Moreover, assembly of the linear systems of equations arising from Galerkin BEM can be farmed out to codes designed for closed surfaces. No modifications nor augmentations of these linear systems is required, nor is user interaction in the form of marking special edges or nodes, see the core Sect. 4 of this article.

We stress this benefit, because it sets our method apart from the heuristics employed in computational acoustics and electromagnetics so far. For these approaches the underlying intuition is that the unknowns of the first-kind BIEs still represent local jumps of field variables. For instance, for the geometric situation of a triple-junction of Fig. 1 (left) and a scalar field, this means that the three jumps have to add to zero at the junction line, which gives rise to an algebraic constraint on the level of boundary-element degrees of freedom. Similarly, for electromagnetic scattering, simulation codes impose a sort of Kirchhoff law at junction lines: the equivalent surface currents have to satisfy some linear constraints, see [30, Section V], [35, Section 3.4], [6, Section 3], and [10, Section I]. Alternatively, in [18] the authors impose essential Kirchhoff conditions in the trial space, while [10] proposes to enforce the Kirchhoff condition weakly on the discrete level in the spirit of mortar finite element techniques. A rigorous mathematical underpinning for these approaches and analysis in suitable trace spaces has not been provided thus far.

\section{List of symbols}

$\mathbb{H}^{+\frac{1}{2}}(\Gamma):=H^{1}\left(\mathbb{R}^{d} \backslash \bar{\Gamma}\right) / H_{0, \Gamma}^{1}\left(\mathbb{R}^{d}\right)$, multi-trace space, (2.2a)

$\mathbb{H}^{-\frac{1}{2}}(\Gamma):=\mathbf{H}\left(\operatorname{div}, \mathbb{R}^{d} \backslash \bar{\Gamma}\right) / \mathbf{H}_{0, \Gamma}\left(\operatorname{div}, \mathbb{R}^{d}\right)$, multi-trace space, $(2.2 \mathrm{~b})$

$H^{+\frac{1}{2}}([\Gamma]):=H^{1}\left(\mathbb{R}^{d}\right) / H_{0, \Gamma}^{1}\left(\mathbb{R}^{d}\right)$, single-trace space, (2.3a)

$H^{-\frac{1}{2}}([\Gamma]):=\mathbf{H}\left(\operatorname{div}, \mathbb{R}^{d}\right) / \mathbf{H}_{0, \Gamma}\left(\operatorname{div}, \mathbb{R}^{d}\right)$, single-trace space, $(2.3 \mathrm{~b})$

$\widetilde{H}^{+\frac{1}{2}}([\Gamma]):=\mathbb{H}^{+\frac{1}{2}}(\Gamma) / H^{+\frac{1}{2}}([\Gamma])$, jump space, $(2.4)$

$\widetilde{H}^{-\frac{1}{2}}([\Gamma]):=\mathbb{H}^{-\frac{1}{2}}(\Gamma) / H^{-\frac{1}{2}}([\Gamma])$, jump space, $(2.4)$

$\ll \dot{u}, \dot{p} \gg:=\int_{[\Gamma]} \dot{u} \dot{p} d \sigma$, bilinear pairing on $\mathbb{H}^{+\frac{1}{2}}(\Gamma) \times \mathbb{H}^{-\frac{1}{2}}(\Gamma),(2.6)$

$\mathbb{H}^{-\frac{1}{2}}\left(\operatorname{curl}_{\Gamma}, \Gamma\right):=\mathbf{H}\left(\mathbf{c u r l}, \mathbb{R}^{3} \backslash \bar{\Gamma}\right) / \mathbf{H}_{0, \Gamma}\left(\mathbf{c u r l}, \mathbb{R}^{3} \backslash \bar{\Gamma}\right)$, multi-trace space, (2.8)

$\mathbf{H}^{-\frac{1}{2}}\left(\operatorname{curl}_{\Gamma},[\Gamma]\right):=\mathbf{H}\left(\mathbf{c u r l}, \mathbb{R}^{3}\right) / \mathbf{H}_{0, \Gamma}\left(\right.$ curl, $\left.\mathbb{R}^{3}\right)$, single-trace space, (2.9)

$\widetilde{\mathbf{H}}^{-\frac{1}{2}}\left(\operatorname{curl}_{\Gamma},[\Gamma]\right):=\mathbb{H}^{-\frac{1}{2}}\left(\operatorname{curl}_{\Gamma}, \Gamma\right) / \mathbf{H}^{-\frac{1}{2}}\left(\operatorname{curl}_{\Gamma},[\Gamma]\right)$, jump space, $(2.10)$

$\ll \dot{\mathbf{u}}, \dot{\mathbf{v}} \gg_{\times}=\int_{[\Gamma]}(\dot{\mathbf{u}} \times \mathbf{n}) \cdot \dot{\mathbf{v}} d \sigma$, bilinear pairing on $\mathbb{H}^{-\frac{1}{2}}\left(\operatorname{curl}_{\Gamma}, \Gamma\right) \times$

$\mathbb{H}^{-\frac{1}{2}}\left(\operatorname{curl}_{\Gamma}, \Gamma\right),(2.11)$

$\operatorname{grad}_{\Gamma}:$ surface gradient, $(2.12)$

$\operatorname{curl}_{\Gamma}:$ surface rotation/curl, (2.13)

$\gamma_{D} / \gamma_{N}$ : Dirichlet/Neumann trace, Page 8

$\mathrm{SL}_{\kappa} / \mathrm{DL}_{\kappa}$ : single-layer and double-layer potentials, Page 8

$\mathrm{V}_{\kappa} / \mathrm{W}_{\kappa}$ : weakly singular and hypersingular BIO, (3.1), (3.2)

$\gamma_{T} / \gamma_{R}$ : electric and magnetic trace, (3.13a) and (3.13b)

$\mathscr{T}_{0}$ : triangulation of screen $\Gamma$, Page 12

$\mathscr{T}$ : triangulation of inflated screen, Page 12 
$\mathscr{S}_{1}^{0}(\mathscr{T})$ p.w. linear continuous functions on inflated screen, Page 14

$\mathscr{S}_{0}^{-1}(\mathscr{T})$ p.w. constant functions on inflated screen, Page 14

\section{Trace spaces: quotient-space view}

We briefly review the new perspective on trace spaces on multi-screens developed in [8, Section 4-6] and [9, Section 3-5]. The underlying ideas will inspire the construction of boundary element spaces in Sect. 4.

\subsection{Acoustic scattering: scalar trace spaces}

Given a multi-screen we consider the following chains of nested Sobolev spaces of functions/vectorfields ${ }^{1}$

$$
\begin{aligned}
& H_{0, \Gamma}^{1}\left(\mathbb{R}^{d}\right) \subset H^{1}\left(\mathbb{R}^{d}\right) \subset H^{1}\left(\mathbb{R}^{d} \backslash \bar{\Gamma}\right), \\
& \mathbf{H}_{0, \Gamma}\left(\operatorname{div}, \mathbb{R}^{d}\right) \subset \mathbf{H}\left(\operatorname{div}, \mathbb{R}^{d}\right) \subset \mathbf{H}\left(\operatorname{div}, \mathbb{R}^{d} \backslash \bar{\Gamma}\right),
\end{aligned}
$$

where a subscript $X_{0, \Gamma}$ indicates a space obtained as the closure in $X$ of smooth functions/vectorfields compactly supported in $\mathbb{R}^{d} \backslash \bar{\Gamma}$. All inclusions in (2.1) define closed subspaces, which renders the associated quotient spaces Hilbert spaces. A particular pair of them, called multi-trace spaces [8, Section 5], is

$$
\begin{aligned}
& \mathbb{H}^{+\frac{1}{2}}(\Gamma):=H^{1}\left(\mathbb{R}^{d} \backslash \bar{\Gamma}\right) / H_{0, \Gamma}^{1}\left(\mathbb{R}^{d}\right), \\
& \mathbb{H}^{-\frac{1}{2}}(\Gamma):=\mathbf{H}\left(\operatorname{div}, \mathbb{R}^{d} \backslash \bar{\Gamma}\right) / \mathbf{H}_{0, \Gamma}\left(\operatorname{div}, \mathbb{R}^{d}\right) .
\end{aligned}
$$

We will tag the elements of these spaces with a dot on top (e.g. $\dot{u}, \dot{p})$, and the symbol under the should be regarded as a representative function $\in H^{1}\left(\mathbb{R}^{d} \backslash \bar{\Gamma}\right)$ or $\mathbf{H}\left(\right.$ div, $\left.\mathbb{R}^{d} \backslash \bar{\Gamma}\right)$, respectively. Another pair of quotient spaces, the single-trace spaces [8, Section 6.1], are defined as

$$
\begin{aligned}
& H^{+\frac{1}{2}}([\Gamma]):=H^{1}\left(\mathbb{R}^{d}\right) / H_{0, \Gamma}^{1}\left(\mathbb{R}^{d}\right), \\
& H^{-\frac{1}{2}}([\Gamma]):=\mathbf{H}\left(\operatorname{div}, \mathbb{R}^{d}\right) / \mathbf{H}_{0, \Gamma}\left(\operatorname{div}, \mathbb{R}^{d}\right) .
\end{aligned}
$$

From [8, Proposition 6.2] we learn that the spaces $H^{+\frac{1}{2}}([\Gamma])$ and $H^{-\frac{1}{2}}([\Gamma])$ are closed subspaces of $\mathbb{H}^{+\frac{1}{2}}(\Gamma)$ and $\mathbb{H}^{-\frac{1}{2}}(\Gamma)$, respectively. This allows us to introduce the jump spaces [8, Section 6.2]

$$
\widetilde{H}^{+\frac{1}{2}}([\Gamma]):=\mathbb{H}^{+\frac{1}{2}}(\Gamma) / H^{+\frac{1}{2}}([\Gamma]) \text { and } \widetilde{H}^{-\frac{1}{2}}([\Gamma]):=\mathbb{H}^{-\frac{1}{2}}(\Gamma) / H^{-\frac{1}{2}}([\Gamma]) .
$$

\footnotetext{
1 See [17, Section 1.1] for an introduction to the relevant Sobolev spaces.
} 
Trace-like operators for functions in $H^{1}\left(\mathbb{R}^{d} \backslash \bar{\Gamma}\right)$ and $\mathbf{H}\left(\right.$ div, $\left.\mathbb{R}^{d} \backslash \bar{\Gamma}\right)$ are supplied by the canonical surjections

$$
\pi_{D}: H^{1}\left(\mathbb{R}^{d} \backslash \bar{\Gamma}\right) \rightarrow \mathbb{H}^{\frac{1}{2}}(\Gamma) \text { and } \pi_{N}: \mathbf{H}\left(\operatorname{div}, \mathbb{R}^{d} \backslash \bar{\Gamma}\right) \rightarrow \mathbb{H}^{-\frac{1}{2}}(\Gamma)
$$

Restricted to $H^{1}\left(\mathbb{R}^{d}\right)$ and $\mathbf{H}\left(\operatorname{div}, \mathbb{R}^{d}\right)$ they give rise to traces onto $H^{+\frac{1}{2}}([\Gamma])$ and $H^{-\frac{1}{2}}([\Gamma])$, respectively.

Remark 2.1 As explained in [8, Section 5.2], if $\Gamma=\partial \Omega, \Omega \subset \mathbb{R}^{d}$ a Lipschitz domain, then then the multi-trace spaces agree with product spaces of traces from inside and outside,

$$
\mathbb{H}^{+\frac{1}{2}}(\Gamma)=H^{\frac{1}{2}}(\partial \Omega) \times H^{\frac{1}{2}}(\partial \Omega) \text { and } \mathbb{H}^{-\frac{1}{2}}(\Gamma)=H^{-\frac{1}{2}}(\partial \Omega) \times H^{-\frac{1}{2}}(\partial \Omega),
$$

whereas the single-traces spaces coincide with the standard trace spaces,

$$
H^{+\frac{1}{2}}([\Gamma])=H^{\frac{1}{2}}(\partial \Omega) \text { and } H^{-\frac{1}{2}}([\Gamma])=H^{-\frac{1}{2}}(\partial \Omega),
$$

and so do the jump spaces:

$$
\widetilde{H}^{+\frac{1}{2}}([\Gamma])=H^{\frac{1}{2}}(\partial \Omega) \text { and } \tilde{H}^{-\frac{1}{2}}([\Gamma])=H^{-\frac{1}{2}}(\partial \Omega) .
$$

Remark 2.2 Let us convey an intuitive grasp of the trace spaces introduced above.

We start with the multi-trace spaces and the observation that $\mathbf{H}\left(\operatorname{div}, \mathbb{R}^{d} \backslash \bar{\Gamma}\right)$ is a space of functions attaining different values on both sides of $\Gamma$. Thus functions in the multi-trace space $\mathbb{H}^{+\frac{1}{2}}(\Gamma)$ are multi-valued on $\Gamma$ : they are given independently on both sides of $\Gamma$. A way to understand this is to imagine an "infinitesimally inflated" screen, see Fig. 3 for a $2 \mathrm{D}$ rendering. Then $\mathbb{H}^{+\frac{1}{2}}(\Gamma)$ can be viewed as a standard Dirichlet trace space on the surface of the inflated screen. The same considerations apply to $\mathbb{H}^{-\frac{1}{2}}(\Gamma)$, where we now deal with normal component traces onto the inflated screen.

The single-trace space $H^{+\frac{1}{2}}([\Gamma])$ is easier to understand: it simply comprises single-valued functions on $\Gamma$. More care has to be taken to arrive at the right interpretation of $H^{-\frac{1}{2}}([\Gamma])$, because we have to fix a local normal $\mathbf{n}$ on $\Gamma$ in order to make sense of a single-valued normal component.

Following $\left[8\right.$, Section 5.1] we introduce a bilinear pairing on $\mathbb{H}^{+\frac{1}{2}}(\Gamma) \times \mathbb{H}^{-\frac{1}{2}}(\Gamma)$ :

$$
\ll \dot{u}, \dot{p} \gg:=\int_{[\Gamma]} \dot{u} \dot{p} d \sigma:=\int_{\mathbb{R}^{d} \backslash \bar{\Gamma}} \mathbf{p} \cdot \nabla u+u \operatorname{div}(\mathbf{p}) d \mathbf{x},
$$

with $u \in H^{1}\left(\mathbb{R}^{d} \backslash \bar{\Gamma}\right)$ and $\mathbf{p} \in \mathbf{H}\left(\operatorname{div}, \mathbb{R}^{d} \backslash \bar{\Gamma}\right)$. According to [8, Prop. 5.1] this pairing induces an isometric duality between $\mathbb{H}^{+\frac{1}{2}}(\Gamma)$ and $\mathbb{H}^{-\frac{1}{2}}(\Gamma)$. From [8, Section 6.2] 

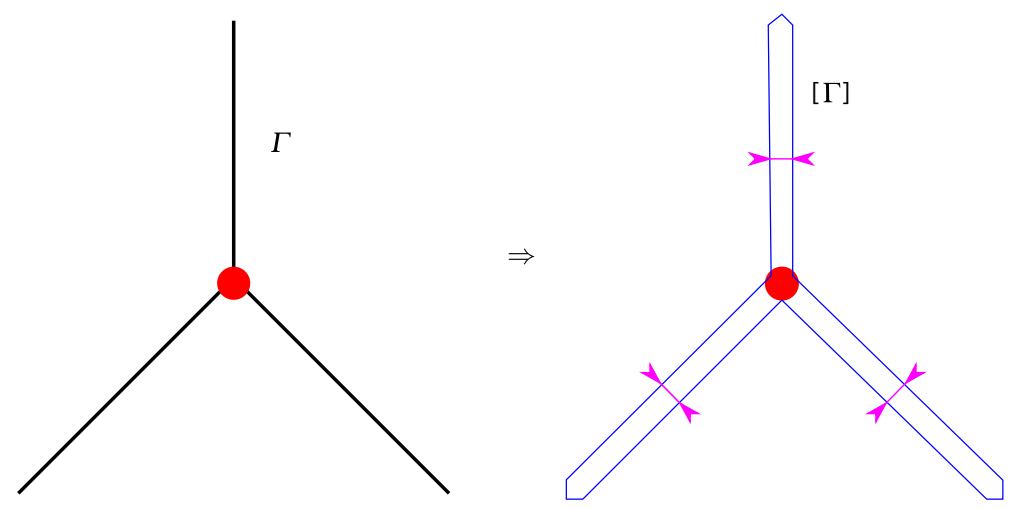

Fig. 3 Inflating a 2D multi-screen

we also learn that $\ll \dot{u}, \dot{p} \gg$ spawns isometric dualities connecting $H^{+\frac{1}{2}}([\Gamma])$ and $\widetilde{H}^{-\frac{1}{2}}([\Gamma])$, and $H^{-\frac{1}{2}}([\Gamma])$ and $\widetilde{H}^{+\frac{1}{2}}([\Gamma])$, respectively.

The bilinear pairing also offers a characterization of single-trace spaces through self-polarity:

Proposition 2.1 ([8, Proposition 6.3]) For $\dot{u} \in \mathbb{H}^{+\frac{1}{2}}(\Gamma)$ and $\dot{p} \in \mathbb{H}^{-\frac{1}{2}}(\Gamma)$ the following equivalences hold true:

$$
\begin{gathered}
\dot{u} \in H^{+\frac{1}{2}}([\Gamma]) \quad \Longleftrightarrow \quad \int_{[\Gamma]} \dot{u} \dot{q} d \sigma=0 \quad \forall \dot{q} \in H^{-\frac{1}{2}}([\Gamma]), \\
\dot{p} \in H^{-\frac{1}{2}}([\Gamma]) \Longleftrightarrow \int_{[\Gamma]} \dot{v} \dot{q} d \sigma=0 \quad \forall \dot{v} \in H^{+\frac{1}{2}}([\Gamma]) .
\end{gathered}
$$

\subsection{Electromagnetic scattering: tangential vectorial trace spaces}

In the context of electromagnetic scattering we start from the chain of nested closed subspaces

$$
\mathbf{H}_{0, \Gamma}\left(\text { curl, } \mathbb{R}^{3}\right) \subset \mathbf{H}\left(\text { curl, } \mathbb{R}^{3}\right) \subset \mathbf{H}\left(\text { curl, } \mathbb{R}^{3} \backslash \bar{\Gamma}\right) .
$$

Parallel to Sect. 2.1 we introduce the quotient spaces of "tangential vector fields"

$$
\begin{array}{ll}
\text { multi-trace space : } & \mathbb{H}^{-\frac{1}{2}}\left(\operatorname{curl}_{\Gamma}, \Gamma\right):=\mathbf{H}\left(\operatorname{curl}, \mathbb{R}^{3} \backslash \bar{\Gamma}\right) / \mathbf{H}_{0, \Gamma}\left(\operatorname{curl}, \mathbb{R}^{3}\right), \\
\text { single-trace space : } & \mathbf{H}^{-\frac{1}{2}}\left(\operatorname{curl}_{\Gamma},[\Gamma]\right):=\mathbf{H}\left(\operatorname{curl}, \mathbb{R}^{3}\right) / \mathbf{H}_{0, \Gamma}\left(\operatorname{curl}, \mathbb{R}^{3}\right), \\
\text { jump space : } \quad \widetilde{\mathbf{H}}^{-\frac{1}{2}}\left(\operatorname{curl}_{\Gamma},[\Gamma]\right):=\mathbb{H}^{-\frac{1}{2}}\left(\operatorname{curl}_{\Gamma}, \Gamma\right) / \mathbf{H}^{-\frac{1}{2}}\left(\operatorname{curl}_{\Gamma},[\Gamma]\right),
\end{array}
$$

with associated canonical surjection $\pi_{T}: \mathbf{H}\left(\operatorname{curl}, \mathbb{R}^{3} \backslash \bar{\Gamma}\right) \rightarrow \mathbb{H}^{-\frac{1}{2}}\left(\operatorname{curl}_{\Gamma}, \Gamma\right)$, which supplies a generalized tangential trace operator. More details can be found in [9, Section 4.3], [9, Def. 4.4], and [9, Def. 4.6], respectively. 
By [9, Prop. 4.2] an isometric self-duality of $\mathbb{H}^{-\frac{1}{2}}\left(\operatorname{curl}_{\Gamma}, \Gamma\right)$ is induced by the bilinear form $\ll \cdot, \cdot \gg_{\times}: \mathbb{H}^{-\frac{1}{2}}\left(\operatorname{curl}_{\Gamma}, \Gamma\right) \times \mathbb{H}^{-\frac{1}{2}}\left(\operatorname{curl}_{\Gamma}, \Gamma\right) \mapsto \mathbb{C}$ defined as

$$
\ll \dot{\mathbf{u}}, \dot{\mathbf{v}} \gg_{\times}:=\int_{[\Gamma]}(\dot{\mathbf{u}} \times \mathbf{n}) \cdot \dot{\mathbf{v}} d \sigma:=\int_{\mathbb{R}^{3} \backslash \bar{\Gamma}} \operatorname{curl}(\mathbf{u}) \cdot \mathbf{v}-\mathbf{u} \cdot \operatorname{curl}(\mathbf{v}) d \mathbf{x},
$$

$\mathbf{u}, \mathbf{v} \in \mathbf{H}\left(\mathbf{c u r l}, \mathbb{R}^{3} \backslash \bar{\Gamma}\right)$. This pairing also gives rise to an isometric duality of $\mathbf{H}^{-\frac{1}{2}}\left(\operatorname{curl}_{\Gamma},[\Gamma]\right)$ and $\widetilde{\mathbf{H}}^{-\frac{1}{2}}\left(\operatorname{curl}_{\Gamma},[\Gamma]\right), c f$. [9, Lemma 4.7]. A result analogous to Proposition 2.1 holds as well:

Proposition 2.2 ( [9, Proposition 4.5]) For $\dot{\mathbf{u}} \in \mathbb{H}^{-\frac{1}{2}}\left(\operatorname{curl}_{\Gamma}, \Gamma\right)$, we have

$$
\dot{\mathbf{u}} \in \mathbf{H}^{-\frac{1}{2}}\left(\operatorname{curl}_{\Gamma},[\Gamma]\right) \Longleftrightarrow \ll \dot{\mathbf{u}}, \dot{\mathbf{v}} \gg_{\times}=0 \quad \forall \dot{\mathbf{v}} \in \mathbf{H}^{-\frac{1}{2}}\left(\operatorname{curl}_{\Gamma},[\Gamma]\right)
$$

The variational formulations of boundary integral equations for electromagnetic scattering rely on surface differential operators. To begin with we define the surface $\operatorname{gradient}_{\operatorname{grad}_{\Gamma}}: \mathbb{H}^{\frac{1}{2}}(\Gamma) \rightarrow \mathbb{H}^{\frac{1}{2}}\left(\operatorname{curl}_{\Gamma}, \Gamma\right)$ through the formula

$$
\operatorname{grad}_{\Gamma}\left(\pi_{D}(p)\right):=\pi_{T}(\operatorname{grad} p) \quad \forall p \in H^{1}\left(\mathbb{R}^{3} \backslash \Gamma\right),
$$

and the surface curl operator $\operatorname{curl}_{\Gamma}: \mathbb{H}^{-\frac{1}{2}}\left(\operatorname{curl}_{\Gamma}, \Gamma\right) \rightarrow \mathbb{H}^{-\frac{1}{2}}(\Gamma)$ using the formula

$$
\operatorname{curl}_{\Gamma}\left(\pi_{T}(\mathbf{u})\right):=\pi_{N}(\operatorname{curl}(\mathbf{u})) \quad \forall \mathbf{u} \in \mathbf{H}\left(\operatorname{curl}, \mathbb{R}^{3} \backslash \bar{\Gamma}\right) .
$$

By restriction and duality the surface differential can also be defined for tangential single-trace and jump spaces, see the commuting diagram of [9, Lemma 5.3].

Remark 2.3 In line with Remark 2.1 we find $\mathbb{H}^{-\frac{1}{2}}\left(\operatorname{curl}_{\Gamma}, \Gamma\right)=\mathbf{H}^{-\frac{1}{2}}\left(\operatorname{curl}_{\Gamma}, \Gamma\right) \times$ $\mathbf{H}^{-\frac{1}{2}}\left(\operatorname{curl}_{\Gamma}, \Gamma\right)$ and $\mathbf{H}^{-\frac{1}{2}}\left(\operatorname{curl}_{\Gamma},[\Gamma]\right)=\mathbf{H}^{-\frac{1}{2}}\left(\operatorname{curl}_{\Gamma}, \Gamma\right)$ for $\Gamma=\partial \Omega, \Omega$ a $3 \mathrm{D}$ Lipschitz domain.

The gist of the interpretation suggested in Remark 2.2 carries over to the vectorial case too.

\section{Boundary integral equations on multi-screens}

We summarize the contents of [8, Section 8] and [9, Section 7-9], which introduced and analyzed representation formulas and boundary integral operators for acoustic and electromagnetic scattering at multi-screens. We restrict ourselves to multi-screens in $3 \mathrm{D}, d=3$.

\subsection{Weakly singular and hypersingular scalar BIEs}

We first study acoustic wave propagation governed by the Helmholtz equation $-\Delta u-$ $\kappa^{2} u=0$ in $\mathbb{R}^{d} \backslash \Gamma, \Gamma$ a multi-screen, $\kappa \in \mathbb{C}, \operatorname{Re} \kappa \geq 0$, the wave number. The two 
relevant trace operators are the ${ }^{2}$

Dirichlet trace:

$$
\gamma_{D}: H^{1}\left(\mathbb{R}^{d} \backslash \Gamma\right) \rightarrow \mathbb{H}^{+\frac{1}{2}}(\Gamma), \quad \gamma_{D}:=\pi_{D},
$$

Neumann trace: $\quad \gamma_{N}: H^{1}\left(\Delta, \mathbb{R}^{d} \backslash \Gamma\right) \rightarrow \mathbb{H}^{-\frac{1}{2}}(\Gamma), \quad \gamma_{N}:=\pi_{N} \circ \operatorname{grad}$,

where we used the canonical surjections from (2.5). By means of two potentials we can state the boundary representation formula [8, (8.3)] for solutions of the homogeneous Helmholtz equation satisfying Sommerfeld radiation conditions. These potentials are the

single-layer potential: $\mathrm{SL}_{\kappa}(\dot{q})(\mathbf{x}):=\int_{[\Gamma]} \gamma_{D}\left(\mathscr{G}_{\kappa, \mathbf{x}}\right) \dot{q} d \sigma, \quad \dot{q} \in \mathbb{H}^{-\frac{1}{2}}(\Gamma)$,

double-layer potential: $\mathrm{DL}_{\kappa}(\dot{v})(\mathbf{x}):=\int_{[\Gamma]} \gamma_{N}\left(\mathscr{G}_{\kappa, \mathbf{x}}\right) \dot{v} d \sigma, \quad \dot{v} \in \mathbb{H}^{+\frac{1}{2}}(\Gamma)$,

where $\mathscr{G}_{\kappa, \mathbf{x}}(\mathbf{y}):=\mathscr{G}_{\kappa}(\mathbf{x}-\mathbf{y})$, with $\mathscr{G}_{\kappa}(\mathbf{z}):=\frac{\exp (\iota \kappa\|\mathbf{z}\|)}{4 \pi\|\mathbf{z}\|}$ being the radiating fundamental solution of the Helmholtz equation in $\mathbb{R}^{3}$.

A key novel feature of the layer potentials for multi-screens are their non-trivial kernels that even allow a precise characterization:

Lemma 3.1 ([8, Lemma 8.6]) The kernels of the layer potentials coincide with the single-trace subspaces:

$$
\begin{aligned}
& \dot{p} \in \mathbb{H}^{-\frac{1}{2}}(\Gamma): \quad \mathrm{SL}_{\kappa}(\dot{p})=0 \Leftrightarrow \dot{p} \in H^{-\frac{1}{2}}([\Gamma]), \\
& \dot{v} \in \mathbb{H}^{-\frac{1}{2}}(\Gamma): \quad \mathrm{DL}_{\kappa}(\dot{v})=0 \quad \Leftrightarrow \quad \dot{v} \in H^{\frac{1}{2}}([\Gamma]) \text {. }
\end{aligned}
$$

By the regularity of the potentials and the pertinent jump relations the following boundary integral operators (BIOs) are well-defined and continuous:

$$
\begin{aligned}
\text { Weakly singular BIO: } & \mathrm{V}_{\kappa}:=\gamma_{D} \circ \mathrm{SL}_{\kappa}: \mathbb{H}^{-\frac{1}{2}}(\Gamma) \rightarrow \mathbb{H}^{+\frac{1}{2}}(\Gamma), \\
\text { Hypersingular BIO: } & \mathrm{W}_{\kappa}:=\gamma_{N} \circ \mathrm{DL}_{\kappa}: \mathbb{H}^{+\frac{1}{2}}(\Gamma) \rightarrow \mathbb{H}^{-\frac{1}{2}}(\Gamma) .
\end{aligned}
$$

For sufficiently regular arguments the weakly singular BIO can be stated in integral form

$$
\left(\mathrm{V}_{\kappa} \dot{\phi}\right)(\mathbf{x})=\int_{[\Gamma]} \mathscr{G}_{\kappa}(\mathbf{x}-\mathbf{y}) \dot{\phi}(\mathbf{y}) d \sigma(\mathbf{y}), \quad \dot{\phi} \in \mathbb{H}^{-\frac{1}{2}}(\Gamma) \cap \mathbb{L}^{\infty}(\Gamma),
$$

where integration is carried out over the virtual inflated screen, $c f$. Fig. 3.

Both integral operators occur in first-kind boundary integral equations (BIE) related to exterior boundary value problems (BVPs) for the Helmholtz equation. If $u \in H_{\mathrm{loc}}^{1}\left(\mathbb{R}^{d} \backslash \Gamma\right)$ is a solution of the exterior Helmholtz Dirichlet BVP

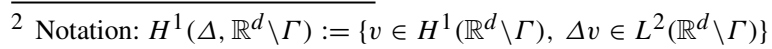




$$
\left\{\begin{array}{llrl}
-\Delta u-\kappa^{2} u & =0 & & \text { in } \mathbb{R}^{d} \backslash \bar{\Gamma}, \\
\gamma_{D} u & =\dot{g}_{D} \in H^{+\frac{1}{2}}([\Gamma]) & \text { on } \Gamma, \\
\lim _{r \rightarrow \infty} r\left(\frac{\partial u}{\partial r}(\mathbf{x})-i \kappa u(\mathbf{x})\right) & =0, & & r:=\|\mathbf{x}\|,
\end{array}\right.
$$

then the unknown Neumann trace $\gamma_{N}(u) \in \mathbb{H}^{-\frac{1}{2}}(\Gamma)$ can be found by solving

$$
\dot{\phi} \in \mathbb{H}^{-\frac{1}{2}}(\Gamma): \quad \mathrm{V}_{\kappa}(\dot{\phi})=\dot{g}_{D}
$$

This BIE can be cast in equivalent variational form as follows: find $\dot{\phi} \in \mathbb{H}^{-\frac{1}{2}}(\Gamma)$ such that

$$
\ll \mathrm{V}_{\kappa} \dot{\phi}, \dot{\psi} \gg=\ll \dot{g}_{D}, \dot{\psi} \gg \quad \forall \dot{\psi} \in \mathbb{H}^{-\frac{1}{2}}(\Gamma)
$$

We can proceed similarly for the exterior Helmholtz-Neumann BVP

$$
\left\{\begin{array}{llrl}
-\Delta u(\mathbf{x})-\kappa^{2} u & =0 & & \text { in } \mathbb{R}^{d} \backslash \bar{\Gamma}, \\
\gamma_{N} u & =\dot{h}_{N} \in H^{-\frac{1}{2}}([\Gamma]) & \text { on } \Gamma, \\
\lim _{r \rightarrow \infty} r\left(\frac{\partial u}{\partial r}(\mathbf{x})-i \kappa u(\mathbf{x})\right) & =0, & & r=\|\mathbf{x}\|,
\end{array}\right.
$$

for which the unknown Dirichlet data $\gamma_{D}(u) \in \mathbb{H}^{+\frac{1}{2}}(\Gamma)$ solve the BIE

$$
\dot{v} \in \mathbb{H}^{+\frac{1}{2}}(\Gamma): \quad \mathrm{W}_{\kappa}(\dot{v})=\dot{h}_{N} .
$$

Also this BIE can be written in variational form and it results in the problem

$$
\text { Find } \dot{v} \in \mathbb{H}^{+\frac{1}{2}}(\Gamma) \text { such that } \quad \ll \mathrm{W}_{\kappa} \dot{v}, \dot{p} \gg=\ll \dot{h}_{N}, \dot{p} \gg \quad \forall \dot{p} \in \mathbb{H}^{+\frac{1}{2}}(\Gamma) \text {. }
$$

The bilinear form on the left-hand side can be conveniently expressed by integration by parts as shown in [31, Section 3.3]. For sufficiently regular argument functions we find the integral representation through an improper integral over the virtual inflated screen:

$$
\begin{aligned}
\ll \mathrm{W}_{\kappa} \dot{v}, \dot{p} \gg= & \int_{[\Gamma]} \int_{[\Gamma]} G_{\kappa}(\mathbf{y}-\mathbf{x})\left\{\left(\operatorname{grad}_{\Gamma} \dot{v} \times \mathbf{n}\right)(\mathbf{y}) \cdot\left(\operatorname{grad}_{\Gamma} \dot{p} \times \mathbf{n}\right)(\mathbf{x})\right. \\
& \left.-\kappa^{2} \mathbf{n}(\mathbf{y}) \cdot \mathbf{n}(\mathbf{y}) \dot{v}(\mathbf{y}) \dot{p}(\mathbf{x})\right\} d \sigma(\mathbf{y}) d \sigma(\mathbf{x})
\end{aligned}
$$

Lemma 3.1 has the direct implication that also the BIOs $\mathrm{V}_{\kappa}$ and $\mathrm{W}_{\kappa}$ have non-trivial kernels given by single-trace functions.

Lemma 3.2 (Kernels of boundary integral operators) The kernels of $V_{\kappa}$ and $W_{\kappa}$ agree with $H^{-\frac{1}{2}}([\Gamma])$ and $H^{+\frac{1}{2}}([\Gamma])$, respectively. 
Hence, $\mathrm{V}_{\kappa}$ and $\mathrm{W}_{\kappa}$ remain well-defined on the quotient spaces $\widetilde{H}^{-\frac{1}{2}}([\Gamma])$ and $\widetilde{H}^{+\frac{1}{2}}([\Gamma])$, respectively. They even enjoy coercivity on jump spaces: there exist compact operators $\mathrm{K}_{V}: \widetilde{H}^{-\frac{1}{2}}([\Gamma]) \rightarrow H^{+\frac{1}{2}}([\Gamma])$ and $\mathrm{K}_{W}: \widetilde{H}^{+\frac{1}{2}}([\Gamma]) \rightarrow H^{-\frac{1}{2}}([\Gamma])$ such that the following Gårding inequalities are satisfied [8, Prop. 8.8]

$$
\begin{array}{cl}
\operatorname{Re}\left\{\int_{[\Gamma]} \dot{q}\left(\mathrm{~V}_{\kappa}+\mathrm{K}_{V}\right) \overline{\dot{q}} d \sigma\right\} \geq C\|\dot{q}\|_{\widetilde{H}^{-\frac{1}{2}}([\Gamma])}^{2} & \forall \dot{q} \in \widetilde{H}^{-\frac{1}{2}}([\Gamma]), \\
\operatorname{Re}\left\{\int_{[\Gamma]} \dot{v}\left(\mathrm{~W}_{\kappa}+\mathrm{K}_{W}\right) \overline{\dot{v}} d \sigma\right\} \geq C\|\dot{v}\|_{\widetilde{H}^{+\frac{1}{2}}([\Gamma])}^{2} \quad \forall \dot{v} \in \widetilde{H}^{+\frac{1}{2}}([\Gamma]),
\end{array}
$$

with $C>0$ depending only on $\kappa$ and $\Gamma$.

We remark that the presence of non-trivial kernels thwarts uniqueness of solutions of (3.6) and (3.9). Yet, Proposition 2.1 still gives us existence, since $\dot{g}_{D} \in H^{+\frac{1}{2}}([\Gamma])$ and $\dot{h}_{N} \in H^{-\frac{1}{2}}([\Gamma])$ ensures consistency of the right-hand side linear forms: they vanish on the single-trace spaces.

\subsection{Electric-field integral equations}

The complex amplitudes of the electric and magnetic fields for time-harmonic electromagnetic waves propagating in empty space satisfy the homogeneous Maxwell's equations curl curlE $-\kappa^{2} \mathbf{E}=\mathbf{0}$, with wave number $\kappa>0$. This second order partial differential equation induces two key trace operators:

$$
\begin{aligned}
\text { Electric trace: } & \gamma_{T}: \mathbf{H}\left(\mathbf{c u r l}, \mathbb{R}^{3} \backslash \bar{\Gamma}\right) \rightarrow \mathbb{H}^{-\frac{1}{2}}\left(\operatorname{curl}_{\Gamma}, \Gamma\right), \quad \gamma_{T}:=\pi_{T}, \quad \text { (3.13a) } \\
\text { Magnetic trace: } & \gamma_{R}: \mathbf{H}\left(\operatorname{curl}^{2}, \mathbb{R}^{3} \backslash \bar{\Gamma}\right) \rightarrow \mathbb{H}^{-\frac{1}{2}}\left(\operatorname{curl}_{\Gamma}, \Gamma\right), \quad \gamma_{R}:=\pi_{T} \circ \text { curl },
\end{aligned}
$$

where $\pi_{T}$ is the canonical surjection implied by the definition (2.8) of $\mathbf{H}^{-\frac{1}{2}}\left(\operatorname{curl}_{\Gamma}, \Gamma\right)$. Both trace operators are continuous and surjective. They are instrumental in the definition of Maxwell single- and double-layer potentials, here given in distributional form as in [9, Section 7.1]: for $\dot{\mathbf{u}} \in \mathbb{H}^{-\frac{1}{2}}\left(\operatorname{curl}_{\Gamma}, \Gamma\right)$,

$$
\begin{aligned}
\mathbf{S L}_{\kappa}(\dot{\mathbf{u}}) & =-\mathscr{G}_{\kappa} * \gamma_{T}^{\prime}(\dot{\mathbf{u}})+\kappa^{-2} \nabla\left(\mathscr{G}_{\kappa} * \gamma_{D}^{\prime} \cdot \operatorname{curl}_{\Gamma}(\dot{\mathbf{u}})\right), \\
\mathbf{D L}_{\kappa}(\dot{\mathbf{u}}) & =-\mathscr{G}_{\kappa} * \gamma_{R}^{\prime}(\dot{\mathbf{u}}) .
\end{aligned}
$$

Slightly abusing notation, the operator $\mathscr{G}_{\kappa} *$ is the Newton potential for the vectorial Helmholtz operator with wave number $\kappa>0$ [31, Section 3.1.1]. From [9, Section 7.2] we know that the single-layer potential $\mathbf{S L}_{\kappa}$ maps continuously the space $\mathbb{H}^{-\frac{1}{2}}\left(\operatorname{curl}_{\Gamma}, \Gamma\right)$ into $\mathbf{H}_{\text {loc }}\left(\mathbf{c u r l}, \mathbb{R}^{3}\right)$ and the double-layer potential $\mathbf{D L} \boldsymbol{L}_{\kappa}$ maps continuously the space $\mathbb{H}^{-\frac{1}{2}}\left(\operatorname{curl}_{\Gamma}, \Gamma\right)$ into $\mathbf{H}_{\text {loc }}\left(\operatorname{curl}, \mathbb{R}^{3} \backslash \bar{\Gamma}\right)$. 
We consider the exterior boundary value problem modeling electromagnetic scattering at the screen $\Gamma$ :

$$
\begin{cases}\operatorname{curl} \operatorname{curl} \mathbf{E}-\kappa^{2} \mathbf{E}=\mathbf{0} & \text { in } \mathbb{R}^{d} \backslash \bar{\Gamma} \\ \gamma_{T} \mathbf{E}=\mathbf{\mathbf { g }} \in \mathbf{H}^{-\frac{1}{2}}\left(\operatorname{curl}_{\Gamma},[\Gamma]\right) & \text { on } \Gamma, \\ \lim _{r \rightarrow \infty} r\left(\operatorname{curl} \mathbf{E}(\mathbf{x}) \times \frac{\mathbf{x}}{\|\mathbf{x}\|}-i \kappa \mathbf{E}(\mathbf{x})\right)=0, & r:=\|\mathbf{x}\| .\end{cases}
$$

Introducing the boundary integral operator $\mathrm{T}_{\kappa}:=\gamma_{T} \circ \mathbf{S L}_{\kappa}: \mathbb{H}^{-\frac{1}{2}}\left(\operatorname{curl}_{\Gamma}, \Gamma\right) \rightarrow$ $\mathbb{H}^{-\frac{1}{2}}\left(\operatorname{curl}_{\Gamma}, \Gamma\right)$ we find that the magnetic trace $\dot{\mathbf{p}}:=\gamma_{R}(\mathbf{E}) \in \mathbb{H}^{-\frac{1}{2}}\left(\operatorname{curl}_{\Gamma}, \Gamma\right)$ of the solution $\mathbf{E}$ of (3.16) solves the first-kind boundary integral equation

$$
\mathrm{T}_{\kappa} \dot{\mathbf{p}}=\dot{\mathbf{g}} \text { in } \mathbb{H}^{-\frac{1}{2}}\left(\operatorname{curl}_{\Gamma}, \Gamma\right),
$$

called the electric field integral equation (EFIE), which can be cast into weak form: Seek $\dot{\mathbf{p}} \in \mathbb{H}^{-\frac{1}{2}}\left(\operatorname{curl}_{\Gamma}, \Gamma\right)$ such that

$$
\ll \mathrm{T}_{\kappa}(\dot{\mathbf{p}}), \dot{\mathbf{q}} \gg_{\times}=\ll \dot{\mathbf{g}}, \dot{\mathbf{q}} \gg_{\times} \quad \forall \dot{\mathbf{q}} \in \mathbb{H}^{-\frac{1}{2}}\left(\operatorname{curl}_{\Gamma}, \Gamma\right)
$$

It is possible to give a more explicit form to the left-hand side of the EFIE by plugging into it the definition of the single layer potential:

$$
\begin{aligned}
& \ll \mathrm{T}_{\kappa}(\dot{\mathbf{p}}), \dot{\mathbf{q}} \gg_{\times} \\
& =\kappa^{-2} \ll \gamma_{D} \cdot \mathscr{G}_{\kappa} * \gamma_{D}^{\prime}\left(\operatorname{curl}_{\Gamma} \dot{\mathbf{p}}\right), \operatorname{curl}_{\Gamma} \dot{\mathbf{q}} \gg-\ll \gamma_{T} \cdot \mathscr{G}_{\kappa} * \gamma_{T}^{\prime}(\dot{\mathbf{p}}), \dot{\mathbf{q}} \gg_{\times},
\end{aligned}
$$

which, for sufficiently regular $\dot{\mathbf{p}}, \dot{\mathbf{q}} \in \mathbb{H}^{-\frac{1}{2}}\left(\operatorname{curl}_{\Gamma}, \Gamma\right)$, can be written explicitly as

$$
\begin{aligned}
& \ll \gamma_{D} \cdot \mathscr{G}_{\kappa} * \gamma_{D}^{\prime}\left(\operatorname{curl}_{\Gamma} \dot{\mathbf{p}}\right), \operatorname{curl}_{\Gamma} \dot{\mathbf{q}} \gg \\
& =\int_{[\Gamma]} \int_{[\Gamma]} \mathscr{G}_{\kappa}(\mathbf{x}-\mathbf{y}) \operatorname{curl}_{\Gamma} \dot{\mathbf{p}}(\mathbf{x}) \operatorname{curl}_{\Gamma} \dot{\mathbf{q}}(\mathbf{y}) d \sigma(\mathbf{x}) d \sigma(\mathbf{y}), \\
& \ll \gamma_{T} \cdot \mathscr{G}_{\kappa} * \gamma_{T}^{\prime}(\dot{\mathbf{p}}), \dot{\mathbf{q}} \gg_{\times} \\
& =\int_{[\Gamma]} \int_{[\Gamma]} \mathscr{G}_{\kappa}(\mathbf{x}-\mathbf{y})(\mathbf{n}(\mathbf{x}) \times \dot{\mathbf{p}}(\mathbf{x})) \cdot(\mathbf{n}(\mathbf{y}) \times \dot{\mathbf{q}}(\mathbf{y})) d \sigma(\mathbf{x}) d \sigma(\mathbf{y}) .
\end{aligned}
$$

The weak EFIE possesses a unique solution in the jump space $\widetilde{\mathbf{H}}^{-\frac{1}{2}}\left(\operatorname{curl}_{\Gamma},[\Gamma]\right)$, since its associated bilinear form satisfies a generalized Gårding inequality, see $[9$, Section 9]. Conversely, solutions in $\mathbb{H}^{-\frac{1}{2}}\left(\operatorname{curl}_{\Gamma}, \Gamma\right)$ cannot be unique:

Lemma 3.3 (Kernel of EFIE boundary integral operator, [9, Lemma 7.9]) The kernel of $T_{\kappa}$ coincides with the single-trace space $\mathbf{H}^{-\frac{1}{2}}\left(\operatorname{curl}_{\Gamma},[\Gamma]\right)$.

Fortunately, as $\dot{\mathbf{g}} \in \mathbf{H}^{-\frac{1}{2}}\left(\operatorname{curl}_{\Gamma},[\Gamma]\right)$, the right-hand side of (3.18) is consistent thanks to Proposition 2.2. 

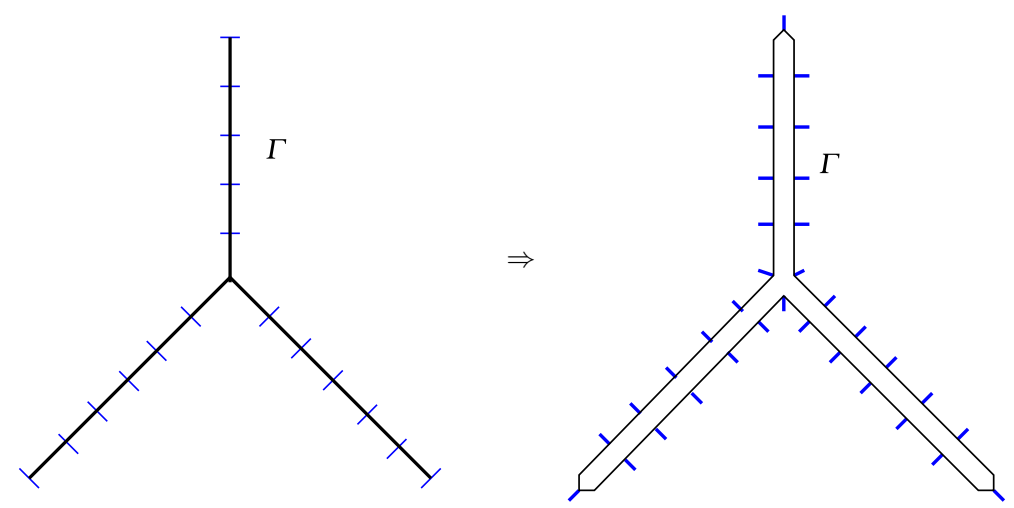

Fig. 4 2D multi-screen and inflated screen equipped with a mesh: the blue strokes represent nodes of the mesh

\section{Quotient-space boundary-element methods}

We aim for a conforming Galerkin discretization of the variational boundary integral equations (3.6), (3.9), and (3.18), employing piecewise polynomial subspaces of the multi-trace spaces $\mathbb{H}^{+\frac{1}{2}}(\Gamma), \mathbb{H}^{-\frac{1}{2}}(\Gamma)$, and $\mathbb{H}^{-\frac{1}{2}}\left(\operatorname{curl}_{\Gamma}, \Gamma\right)$.

Functions belonging to multi-traces spaces can have different values on "opposite sides" of parts of a multi-screen. In the spirit of Remark 2.2 we adopt the perspective of a virtual inflated screen as indicated in Fig. 3 for a 2D situation. On such an inflated screen $[\Gamma]$, in a combinatorial sense, a "virtual surface mesh" $\mathscr{T}$ consisting of smooth panels can be defined as if $[\Gamma]$ was the surface of a domain, see Fig. 4 right. In terms of geometry, different panels may overlap or even coincide, of course.

We restrict ourselves to triangulated multi-screens embedded in 3D space as already adressed in the Introduction. For the sake of simplicity we assume that the multi-screen $\Gamma$ is composed of flat parts only and that all meshes comprise only flat triangular panels.

\subsection{Oriented multi-screen surface triangulations}

Let $\mathscr{T}_{0}$ be a triangulation of $\Gamma$, that is, a set of open flat triangles, $\mathscr{T}_{0}=\{K\}$, such that

(I) $\bar{\Gamma}_{0}=\bigcup\left\{\bar{K}: K \in \mathscr{T}_{0}\right\}$,

(II) the triangles $K$ are mutually disjoint: $K, K^{\prime} \in \mathscr{T}_{0}, K \neq K^{\prime}$ implies $K \cap K^{\prime}=\emptyset$,

(III) for $K, K^{\prime} \in \mathscr{T}_{0}, K \neq K^{\prime}$, the intersection $\bar{K} \cap \overline{K^{\prime}}$ is either empty or a common vertex or edge of both,

(IV) and no triangle of $\mathscr{T}_{0}$ has more than one edge on the boundary $\partial \Gamma$.

The notion of "edges", "boundary edges", and "nodes" of $\mathscr{T}_{0}$ should be clear. Requirement (IV) has been included merely to simplify the presentation of the algorithm below. Further, we designate

- by $\mathscr{E}(K)$ the set of the three edges of a triangle $K \in \mathscr{T}_{0}$,

- and by $\mathscr{T}(e)$ the set of triangles abutting an edge $e$ of $\mathscr{T}_{0}$. 


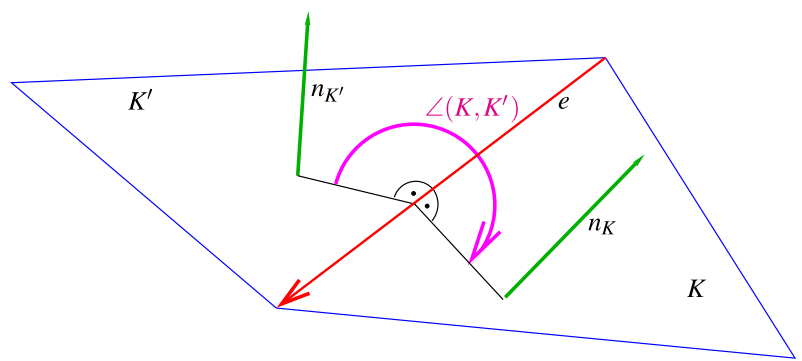

Fig. 5 Definition of the angle between two (oriented) triangles sharing an edge: case of consistently oriented triangles

We equip every triangle $K$ with a fixed orientation by ordering its vertices or, equivalently, prescribing a unit normal vector $\boldsymbol{n}_{K} \in \mathbb{R}^{3}$. We also endow every edge of $\mathscr{T}_{0}$ with an intrinsic direction and write $o_{K, e} \in\{-1,+1\}$ for the relative orientation of the edge $e \in \mathscr{E}(K)$ and the triangle $K$.

For two adjacent triangles $K, K^{\prime} \in \mathscr{T}_{0}$ with joint edge $e:=\partial K \cap \partial K^{\prime}$ we set $o_{K, K^{\prime}}=-o_{K, e} \cdot o_{K^{\prime}, e}$, that is $o_{K, K^{\prime}}=1$ tells us that both triangles are oriented consistently. Then we can define the angle enclosed by $K$ and $K^{\prime}, \angle\left(K, K^{\prime}\right) \in[0,2 \pi)$, as the angle of the counterclockwise rotation around the common edge $\partial K \cap \partial K^{\prime}$ that transforms $o_{K, K^{\prime}} \cdot \boldsymbol{n}_{K^{\prime}}$ into $-\boldsymbol{n}_{K}$, see Fig. 5 .

In a first step for every $K \in \mathscr{T}_{0}$ we create two copies $K^{+}$and $K^{-}$with the same geometry but to be regarded as different entities. The reader may imagine $K^{+}$and $K^{-}$as the two sides of $K$ with $\boldsymbol{n}_{K}$ pointing from $K^{-}$to $K^{+}$. These sides form the set underlying what we call the virtual surface mesh for $\Gamma$ :

$$
\mathscr{T}:=\left\{K^{+}, K^{-}: K \in \mathscr{T}_{0}\right\}
$$

In addition, $K^{+}$will be endowed with the unit normal $\boldsymbol{n}_{K}$, whereas the unit normal $-\boldsymbol{n}_{K}$ is assigned to $K^{-}$. This defines the orientation for every triangle of $\mathscr{T}$.

Now we present an algorithm that constructs the incidence information for $\mathscr{T}$ in the form of the symmetric adjacency relation $\operatorname{adj}_{\mathscr{T}} \subset \mathscr{T} \times \mathscr{T}$ for $\mathscr{T}:\left(K_{1}, K_{2}\right) \in \operatorname{adj}_{\mathscr{T}}$, if and only if these two triangles have a common edge in $\mathscr{T}$. With $\operatorname{adj}_{\mathscr{T}}$ at our disposal the edge and vertex sets for $\mathscr{T}$ can be built.

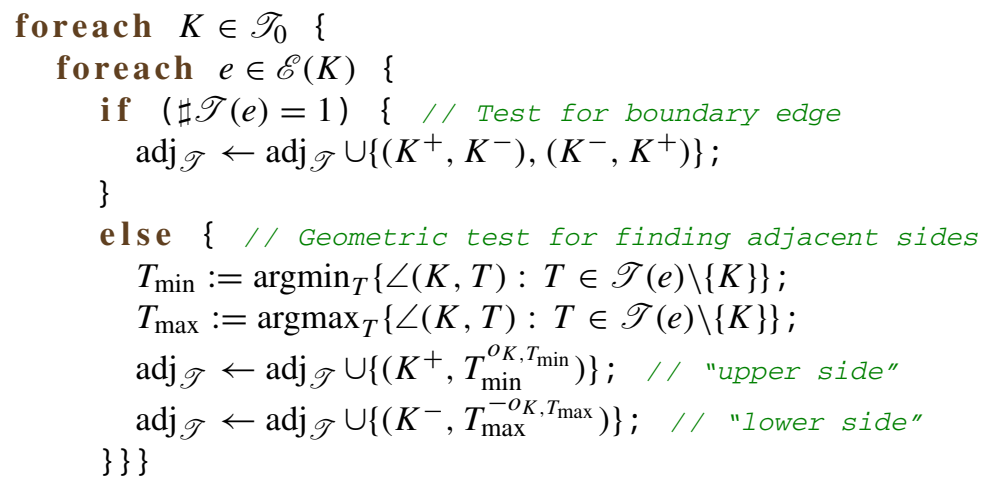


The asymptotic computational effort for running this algorithm is $O\left(\sharp \mathscr{T}_{0}\right)$ for $\sharp \mathscr{T}_{0} \rightarrow \infty$. It yields a relation $\operatorname{adj}_{\mathscr{T}}$ such that

$$
\sharp\left\{\left(T, T^{\prime}\right): \exists T^{\prime} \in \mathscr{T},\left(T, T^{\prime}\right) \in \operatorname{adj}_{\mathscr{T}}\right\}=3 \quad \forall T \in \mathscr{T},
$$

that is, every triangle has three neighbors and every edge of $\mathscr{T}$ belongs to two triangles. This is characteristic of a triangulation of the closed surface of a volume domain. Furthermore, using the normal directions for panels of $\mathscr{T}$ as detailed above, those turn out to be oriented consistently: their normals all point into the exterior of the virtual inflated screen.

\subsection{Boundary-element spaces}

We take for granted the availability of a triangular virtual surface mesh $\mathscr{T}$ as built in Sect. 4.1. On this mesh we introduce the standard lowest-order piecewise polynomial boundary element spaces

- $\mathscr{S}_{1}^{0}(\mathscr{T}) \subset C^{0}([\Gamma])$ of $\mathscr{T}$-piecewise linear "continuous" functions on the inflated screen $[\Gamma]$, and

$-\mathscr{S}_{0}^{-1}(\mathscr{T}) \subset L^{2}([\Gamma])$ of $\mathscr{T}$-piecewise constant functions on $[\Gamma]$.

We equip these spaces with the usual minimally supported local basis functions

$$
\begin{array}{cl}
\mathscr{S}_{1}^{0}(\mathscr{T})=\operatorname{span}\left\{b^{i}\right\}_{i=1}^{N_{V}(\mathscr{T})}, & N_{V}(\mathscr{T}) \hat{=} \text { no. of nodes of } \mathscr{T}, \\
\mathscr{S}_{0}^{-1}(\mathscr{T})=\operatorname{span}\left\{\beta^{i}\right\}_{i=1}^{N_{T}(\mathscr{T})}, & N_{T}(\mathscr{T}) \hat{=} \text { no. of triangles of } \mathscr{T} .
\end{array}
$$

These spaces supply finite-dimensional subspaces of the multi-trace spaces:

$$
\mathscr{S}_{1}^{0}(\mathscr{T}) \subset \mathbb{H}^{+\frac{1}{2}}(\Gamma), \quad \mathscr{S}_{0}^{-1}(\mathscr{T}) \subset \mathbb{H}^{-\frac{1}{2}}(\Gamma),
$$

which qualifies them as trial and test spaces for boundary element Galerkin discretization of the variational problems (3.9) and (3.6), respectively.

For the Galerkin discretization of the EFIE (3.18) we rely on the standard edge element space on $\mathscr{T}[5$, Section 8]

$$
\mathscr{N}_{0}(\mathscr{T}) \subset \mathbb{H}^{-\frac{1}{2}}\left(\operatorname{curl}_{\Gamma}, \Gamma\right)
$$

also known as Rao-Wilton-Glisson (RWG) boundary element space in computational engineering. For the edge-associated local basis functions with minimal supports we write $\eta^{1}, \ldots, \eta^{N_{E}(\mathscr{T})}$, where $N_{E}(\mathscr{T})$ is the total number of edges of $\mathscr{T}$.

These boundary element spaces enjoy the customary approximation properties. In particular, they are asymptotically dense. To state the result, we consider a uniformly shape-regular sequence $\left\{\mathscr{T}_{\ell}\right\}_{\ell \in \mathbb{N}}$ of meshes with $h_{\ell} \rightarrow 0$ for $\ell \rightarrow \infty$, where $h_{\ell}$ stands for the mesh width $h_{\ell}:=\max _{K \in \mathscr{T}_{\ell}} \operatorname{diam} K$. 
Lemma 4.1 (Asymptotic density of boundary element spaces)

$$
\begin{gathered}
\forall \dot{v} \in \mathbb{H}^{+\frac{1}{2}}(\Gamma): \inf _{v_{h} \in \mathscr{S}_{1}^{0}\left(\mathscr{T}_{\ell}\right)}\left\|\dot{v}-v_{h}\right\|_{\mathbb{H}^{+\frac{1}{2}}(\Gamma)} \rightarrow 0, \\
\forall \dot{\varphi} \in \mathbb{H}^{-\frac{1}{2}}(\Gamma): \inf _{\varphi_{h} \in \mathscr{S}_{0}^{-1}\left(\mathscr{T}_{\ell}\right)}\left\|\dot{\varphi}-\varphi_{h}\right\|_{\mathbb{H}^{-\frac{1}{2}(\Gamma)}} \rightarrow 0, \quad \text { for } \ell \rightarrow \infty . \\
\forall \dot{\mathbf{p}} \in \mathbb{H}^{-\frac{1}{2}}\left(\operatorname{curl}_{\Gamma}, \Gamma\right): \inf _{\mathbf{p}_{h} \in \mathscr{N}_{0}\left(\mathscr{T}_{\ell}\right)}\left\|\dot{\mathbf{p}}-\mathbf{p}_{h}\right\|_{\mathbb{H}^{-\frac{1}{2}}\left(\operatorname{curl}_{\Gamma}, \Gamma\right)} \rightarrow 0
\end{gathered}
$$

The proof relies on the fact that, using the notations of Definition 1.2, the space

$$
X^{\infty}:=\left\{v \in C^{\infty}\left(\mathbb{R}^{3} \backslash \Gamma\right), v_{\mid \Omega_{j}} \in C^{\infty}\left(\bar{\Omega}_{j}\right)\right\}
$$

is dense in $H^{1}\left(\mathbb{R}^{3} \backslash \bar{\Gamma}\right)$, and that $\left(X^{\infty}\right)^{3}$ is dense in both $\mathbf{H}\left(\operatorname{div}, \mathbb{R}^{3} \backslash \bar{\Gamma}\right)$ and $\mathbf{H}\left(\mathbf{c u r l}, \mathbb{R}^{3} \backslash \bar{\Gamma}\right)$. Then standard approximation estimates for traces of smooth functions yield asymptotic density.

Remark 4.1 Our considerations can easily be extended to boundary element spaces of higher polynomial degree. We do not elaborate on this just for the sake of a concise presentation.

\subsection{Assembly of BE Galerkin matrices}

We explain the approach in the case of (3.9) for $\operatorname{Re} \kappa \geq 0$ using $\mathscr{S}_{1}^{0}(\mathscr{T})$ as trial and test space. Invoking (3.10) the entries of the Galerkin matrix $\mathbf{A}_{W \kappa} \in \mathbb{C}^{N_{V}(\mathscr{T}), N_{V}(\mathscr{T})}$ are

$$
\begin{aligned}
\left(\mathbf{A}_{W, \kappa}\right)_{k, \ell}= & \sum_{K_{1} \in \mathscr{T}} \sum_{K_{2} \in \mathscr{T}} \int_{K_{1}} \int_{K_{2}} G_{\kappa}(\mathbf{y}-\mathbf{x})\left\{\operatorname{grad}_{\Gamma} b^{k}(\mathbf{y}) \times \mathbf{n}(\mathbf{y}) \cdot \operatorname{grad}_{\Gamma} b^{\ell}(\mathbf{x}) \times \mathbf{n}(\mathbf{x})\right. \\
& \left.-\kappa^{2} \mathbf{n}(\mathbf{y}) \cdot \mathbf{n}(\mathbf{y}) b^{k}(\mathbf{y}) b^{\ell}(\mathbf{x})\right\} d \sigma(\mathbf{y}) d \sigma(\mathbf{x}),
\end{aligned}
$$

for $1 \leq k, \ell \leq N_{V}$, where $b^{k}, b^{\ell}$ are "tent basis functions" of $\mathscr{S}_{1}^{0}(\mathscr{T})$. Note that $\mathbf{n}(\mathbf{y})$ and $\mathbf{n}(\mathbf{x})$ stand for the "exterior" unit normals on $K_{2}$ and $K_{1}$, respectively, as introduced above. The Galerkin matrices $\mathbf{A}_{V, \kappa} \in \mathbb{C}^{N_{T}(\mathscr{T}), N_{T}(\mathscr{T})}$ and $\mathbf{A}_{\mathbf{T}, \kappa} \in \mathbb{C}^{N_{E}(\mathscr{T}), N_{E}(\mathscr{T})}$ for the variational weakly singular BIE (3.6) and EFIE (3.18) are given by analogous formulas based on (3.3) and (3.20), (3.21). We skip the details.

Remark 4.2 The integrals in (4.6) are standard weakly singular integrals over pairs of panels. They can be evaluated using the established quadrature policy from [31, Chapter 5]. If BEM software that can compute contributions of pairs of panels to Galerkin BEM matrices is available, it can be used without further adaption. The only requirement is that the result, up to the last digit, depends exclusively on the geometry of $K_{1}$ and $K_{2}$ and in no way on their internal representation (like the ordering of vertices, etc.). If this condition is not met, one might arrive at linear systems of equations that are not consistent. This will disrupt the convergence of iterative solvers. 


\subsection{Kernels of discretized boundary integral operators}

According to Lemma 3.2 the kernels of the weakly singular and hypersingular boundary integral operators $\mathrm{V}_{\kappa}$ and $\mathrm{W}_{\kappa}$ coincide with single-trace spaces. We immediately conclude that

$$
\begin{aligned}
\operatorname{kern}\left(\mathbf{A}_{W, \kappa}\right) & \longleftrightarrow Z_{W}(\mathscr{T}):=\mathscr{S}_{1}^{0}(\mathscr{T}) \cap H^{+\frac{1}{2}}([\Gamma]) \\
\operatorname{kern}\left(\mathbf{A}_{V, \kappa}\right) & \longleftrightarrow Z_{V}(\mathscr{T}):=\mathscr{S}_{0}^{-1}(\mathscr{T}) \cap H^{-\frac{1}{2}}([\Gamma]) \\
\operatorname{kern}\left(\mathbf{A}_{\mathbf{T}, \kappa}\right) & \longleftrightarrow Z_{\mathbf{T}}(\mathscr{T}):=\mathscr{N}_{0}(\mathscr{T}) \cap \mathbf{H}^{-\frac{1}{2}}\left(\operatorname{curl}_{\Gamma},[\Gamma]\right)
\end{aligned}
$$

Here $\longleftrightarrow$ means that the nullspace of the matrix on the left consists of the vectors of basis expansion coefficients of all functions belonging to the BE function space on the right.

In light of the interpretation of the single-trace spaces as spaces of "uni-valued traces" we find that these kernels are the span of locally supported basis functions associated with the non-inflated screen mesh $\mathscr{T}_{0}$. Writing $N_{V}\left(\mathscr{T}_{0}\right), N_{E}\left(\mathscr{T}_{0}\right)$, and $N_{T}\left(\mathscr{T}_{0}\right)$ for the number of nodes, edges, and triangles in $\mathscr{T}_{0}$, respectively, we conclude

$$
\begin{aligned}
\operatorname{dim} \operatorname{kern}\left(\mathbf{A}_{W, \kappa}\right) & =N_{V}\left(\mathscr{T}_{0}\right), \\
\operatorname{dim} \operatorname{kern}\left(\mathbf{A}_{V, \kappa}\right) & =N_{T}\left(\mathscr{T}_{0}\right), \\
\operatorname{dim} \operatorname{kern}\left(\mathbf{A}_{\mathbf{T}, \kappa}\right) & =N_{E}\left(\mathscr{T}_{0}\right) .
\end{aligned}
$$

Remark 4.3 If the multi-screen consists of a few flat parts, some contributions to the kernels of the Galerkin matrices can be identified easily. Let us examine $\mathscr{S}_{1}^{0}(\mathscr{T}) \cap$ $H^{+\frac{1}{2}}([\Gamma])$ : Let $k$ and $\ell$ be the indices of those two distinct nodes of $\mathscr{T}$ spawned by a single node of $\mathscr{T}_{0}$. Then,

$$
b^{k}+b^{\ell} \in H^{+\frac{1}{2}}([\Gamma]), \quad b^{k}-b^{\ell} \notin H^{+\frac{1}{2}}([\Gamma]) .
$$

Thus, for the sake of Galerkin discretization of the hypersingular BIE, we can replace the two basis functions $b^{k}$ and $b^{\ell}$ by their difference $b^{k}-b^{\ell}$ in the boundary element space. Similarly, if a node of $\mathscr{T}_{0}$ lies on $\partial \Gamma$ and, therefore, spawns only a single node of $\mathscr{T}$ with index $m$, then $b^{m} \in H^{+\frac{1}{2}}([\Gamma])$ and this basis function can be dropped altogether. This results in a reduced boundary element space visualized in Fig. 6 for a $2 \mathrm{D}$ setting.

Remark 4.4 In the case of a simple screen, that is, if $\Gamma$ is an orientable, twodimensional, triangulated manifold with boundary, a solution obtained by the quotient-space BEM agrees with the solution produced by the standard Galerkin BEM in jump space up to a multi-trace function in the kernel of the underlying boundary integral operators. Thus, the quotient-space BEM is a genuine generalization to complex screens of well-established and widely used BEM. 

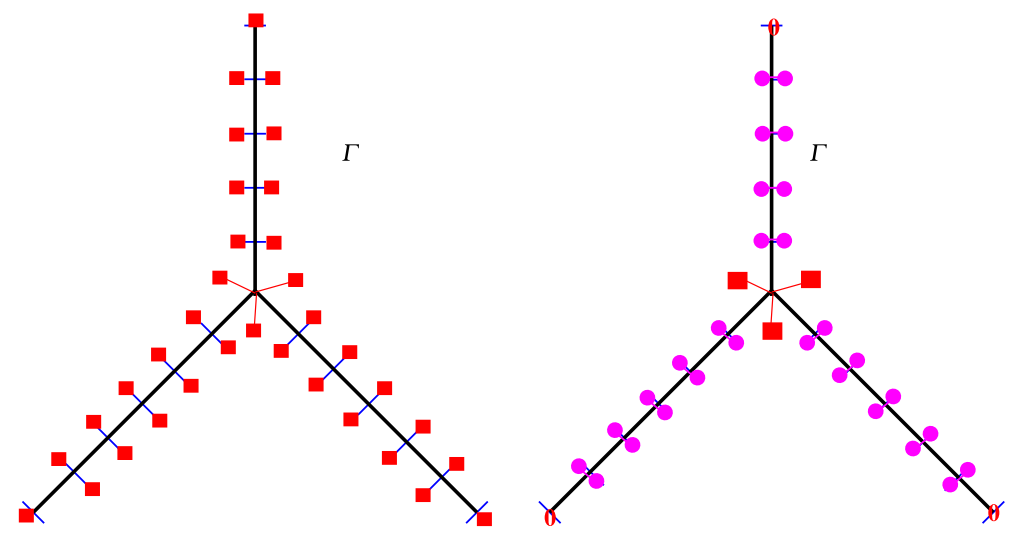

Fig. 6 Reduced boundary element space on $\mathscr{T}$ by eliminating certain functions in $\mathscr{S}_{1}^{0}(\mathscr{T}) \cap H^{+\frac{1}{2}}([\Gamma])$. Red squares represent (retained) basis functions of $\mathscr{S}_{1}^{0}(\mathscr{T})$, magenta bullets differences of basis functions

\subsection{Convergence of Galerkin solutions}

From [8, Prop. 8.9] we learn that the variational boundary integral equations (3.6) and (3.9) have unique solutions in $\widetilde{H}^{-\frac{1}{2}}([\Gamma])$ and $\widetilde{H}^{+\frac{1}{2}}([\Gamma])$, respectively. The analogous result for (3.18) is given in [9, Prop. 6.6] and claims uniqueness of solutions in $\widetilde{\mathbf{H}}^{-\frac{1}{2}}\left(\operatorname{curl}_{\Gamma},[\Gamma]\right)$.

Uniqueness of solutions combined with the coercivity estimates (3.11) and (3.12) paves the way for using the abstract result of [31, Theorem 4.2.9] in the jump spaces $\widetilde{H}^{-\frac{1}{2}}([\Gamma])$ and $\widetilde{H}^{+\frac{1}{2}}([\Gamma])$. Thus we conclude asymptotic quasi-optimality of Galerkin solutions with respect to the norms of the jump spaces.

For instance, if we adopt the setting of Lemma 4.1 and write $\dot{\phi} \in \mathbb{H}^{-\frac{1}{2}}(\Gamma) / \phi_{\ell} \in$ $\mathscr{S}_{0}^{-1}\left(\mathscr{T}_{\ell}\right)$ for the solution/boundary element Galerkin solution of (3.6), then for sufficiently large $\ell$

$$
\begin{aligned}
\left\|\dot{\phi}-\phi_{\ell}\right\|_{\widetilde{H}^{-\frac{1}{2}}([\Gamma])} \leq C \inf _{\varphi_{\ell} \in \mathscr{S}_{0}^{-1}\left(\mathscr{T}_{\ell}\right)}\left\|\dot{\phi}-\varphi_{\ell}\right\|_{\widetilde{H}^{-\frac{1}{2}}([\Gamma])} \\
\leq C \inf _{\varphi_{\ell} \in \mathscr{S}_{0}^{-1}\left(\mathscr{T}_{\ell}\right)}\left\|\dot{\phi}-\varphi_{\ell}\right\|_{\mathbb{H}^{-\frac{1}{2}}(\Gamma)},
\end{aligned}
$$

with $C>0$ independent of $\ell$. A corresponding estimate holds true for (3.9).

For the Galerkin discretization of the variational EFIE (3.18) the situation is more complicated, since the sesqui-linear form merely satisfies a generalized Gårding inequality. Thus, we have to resort to Hodge-type splittings of $\mathbb{H}^{-\frac{1}{2}}\left(\operatorname{curl}_{\Gamma}, \Gamma\right)$ induced by regular decompositions of $\mathbf{H}\left(\right.$ curl, $\mathbb{R}^{3} \backslash \bar{\Gamma}$ ), see [9, Section 9.2]. They possess discrete counterparts and those can be used to verify asymptotic quasi-optimality of Galerkin solutions in $\widetilde{\mathbf{H}}^{-\frac{1}{2}}\left(\operatorname{curl}_{\Gamma},[\Gamma]\right)$, as elaborated in [5, Section 9.1], [2, Section 6], and [3]. 


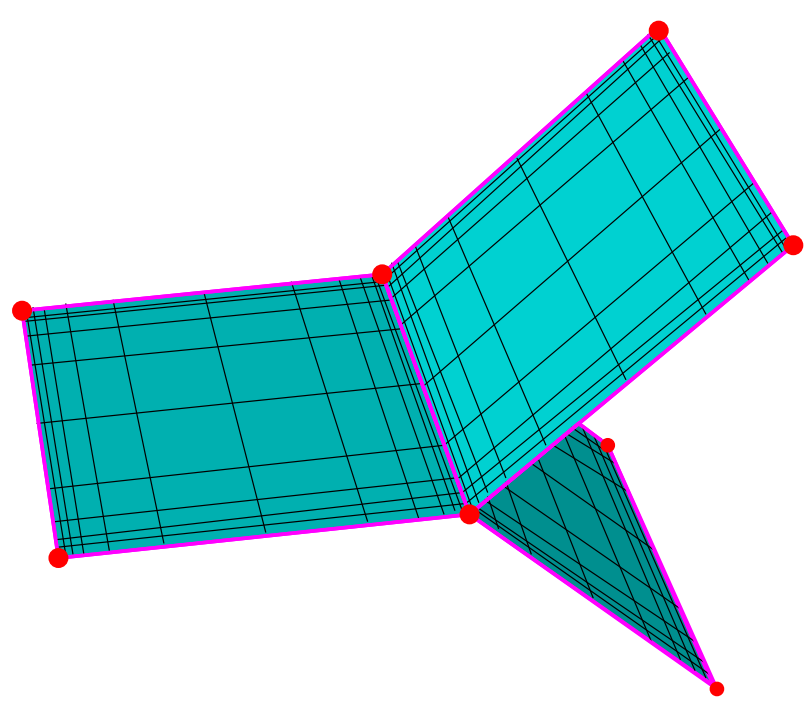

Fig. 7 Simple 3D multi-screen composed of flat polygonal panels (cyan) with highlighted junction line (orange), edges (magenta), and corner points (red). Panels triangulated with algebraically graded tensorproduct mesh

Eventually, quasi-optimality estimates like (4.9) can be combined with interpolation error estimates for boundary element spaces, e.g., from [31, Section 4.1] and [5, Section 8], to infer rates of asymptotic convergence for our boundary element Galerkin solutions in jump-space norms. For instance, for the concrete case of (4.9), a piecewise smooth multi-screen, a shape-regular and quasi-uniform family of meshes $\left\{\mathscr{T}_{\ell}\right\}_{\ell \in \mathbb{N}}$ with meshwidths $h_{\ell}>0$, and assuming that the solution $u$ of (3.4) belongs to $H_{\mathrm{loc}}^{2}\left(\mathbb{R}^{d}\right)$, [31, Theorem 4.1.33] gives us

$$
\left\|\dot{\phi}-\phi_{\ell}\right\|_{\widetilde{H}^{-\frac{1}{2}}([\Gamma])} \leq C h_{\ell}^{3 / 2} \forall \ell \in \mathbb{N}
$$

because $\dot{\phi}$ will belong to $H^{1}$ locally on each smooth part of $\Gamma$.

Remark 4.5 The right-hand side bound in (4.9) can be controlled by choosing meshes adapted to the exact solution $\dot{\phi} \in \mathbb{H}^{-\frac{1}{2}}(\Gamma)$. Since $\dot{\phi}=\gamma_{N}(u)$, where $u \in H_{\mathrm{loc}}^{1}\left(\Delta, \mathbb{R}^{d} \backslash \Gamma\right)$ solves the exterior Dirichlet problem (3.4), elliptic regularity theory for linear scalar second-order boundary value problems, see the monographs [13], [23,25, Chapter $10 \& 11]$, provides precise information on the local behavior of $\dot{\phi}$ at edges, corners, and junction lines of a multi-screen.

Let us discuss the case of a 3D multi-screen $\Gamma$ comprised of a few flat polygonal panels, like that shown in Fig. 7. Assume that the Dirichlet data $\dot{g}_{D} \in H^{+\frac{1}{2}}([\Gamma])$ are the restriction to $\Gamma$ of a function analytic in a neighborhood of $\Gamma$. Then the results published in [11,12,27,29,32] [34, Section 1.5] tell us that

$-\dot{\phi}$ is analytic in the interior of panels of $\Gamma$, 
- in a neighborhood $U \subset[\Gamma]$ of any interior point of a (straight) edge of the screen $\dot{\phi}$ features a $1 / \sqrt{ }$-type singularity,

$$
\dot{\phi}(\mathbf{x})=\frac{1}{\sqrt{d(\mathbf{x}, \partial \Gamma)}} \psi(\mathbf{x})+\eta(\mathbf{x}), \quad \mathbf{x} \in U,
$$

with smooth functions $\psi, \eta$. Here we wrote $d(\mathbf{x}, \partial \Gamma)$ for the distance of $\mathbf{x} \in \Gamma$ to $\partial \Gamma$.

- locally at interior points of junction lines $\dot{\phi}$ is the sum of a smooth function and countably many singular contributions behaving like $r \mapsto r^{\alpha_{k}}, \alpha_{k}>-\frac{1}{2}, \kappa \in \mathbb{N}$, where $r$ is the distance to the junction line,

_ "weaker" singularities arise at corner points.

As pointed out in [16], for low-order BEM this a priori knowledge of the singularities of $\dot{\phi}$ suggests the use of sequences of anisotropic tensor-product meshes algebraically graded towards edges and junction lines with a grading factor $\geq 3$, see Fig. 7. Thus, we can expect to recover the same asymptotic rates of algebraic convergence (in terms of number of degrees of freedom) as for a smooth $\dot{\phi}$ [26, Chapter 3], [28, Section 3], [19, Chapter 7].

If one is not confined to $\mathrm{BE}$ spaces of fixed low polynomial degree, then $h p$ BEM on anisotropic geometrically graded tensor-product meshes is an option offering asymptotic exponential convergence [1,19, Chapter 8]. Since these topics are outside the intended scope of this article, we are not going to pursue them further, neither here nor in the next section.

\section{Numerical results for triangulated multi-screens}

We investigate the performance of quotient-space BEM in a few numerical experiments, which were carried out using the BETL library [21]. For each of the BIE we report the dimensions of the discrete kernels, we compute the generalized condition numbers of the Galerkin matrices (quotient of largest and smallest non-zero singular values), and study the convergence of the Conjugate Gradient (GC) and Generalized Minimal Residual (GMRES) iterative solvers. We stop the iterations once the Euclidean norm of the residual has shrunk by a factor of $10^{6}$.

The experiments were carried out for the multi-screens displayed in Fig. 2: a "triple junction" and a "quadruple junction". That figure also displays the coarsest mesh in each case. Table 1 provides information on the screen mesh $\mathscr{T}_{0}$ and the associated virtual surface mesh $\mathscr{T}$ on different refinement levels. These refinement levels were generated by uniform refinement of $\mathscr{T}_{0}$. As before, $N_{T}(\mathscr{M}), N_{E}(\mathscr{M})$ and $N_{V}(\mathscr{M})$ denote the number of triangular panels, edges, and nodes, respectively, of the screen triangulation $\mathscr{M} \in\left\{\mathscr{T}, \mathscr{T}_{0}\right\}$.

For each of the BIEs of interest, we summarize our results in a table and provide a plot of singular values of the resulting Galerkin matrices for the sequences of meshes. The tables report, for each refinement level (Ref. Level): the number of degrees of freedom (DoFs); the generalized condition number of the Galerkin matrices (Gen. 


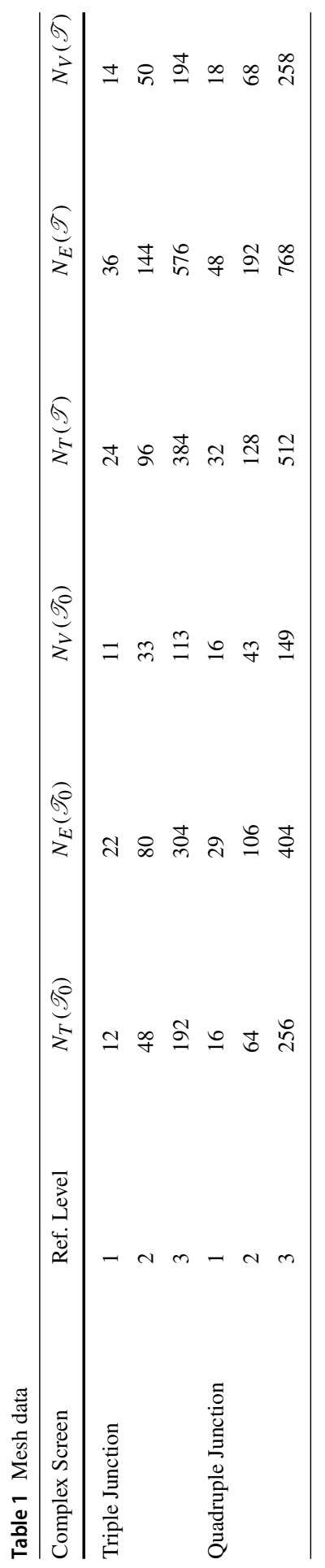


Table 2 Comparison of condition numbers for $\mathbf{A}_{V, 0}, \mathbf{A}_{W, 0}$ and $\mathbf{A}_{T, 1}$ on unit disk discretized with standard $\operatorname{BEM}\left(\mathscr{T}_{0}\right)$ and quotient-space $\operatorname{BEM}(\mathscr{T})$

\begin{tabular}{llllll}
\hline & Ref. Level & \multicolumn{2}{l}{ Standard } & BEM $(\mathscr{T})$ & \multicolumn{2}{l}{ QuotiENT- SPACE BEM $(\mathscr{T})$} \\
& & DoFs & Condition Number & DoFs & Gen. Condition Number \\
\hline $\mathbf{A}_{V, 0}$ & 1 & 20 & $1.52 \cdot 10^{1}$ & 40 & $1.30 \cdot 10^{1}$ \\
& 2 & 80 & $3.84 \cdot 10^{1}$ & 160 & $2.89 \cdot 10^{1}$ \\
& 3 & 320 & $8.37 \cdot 10^{1}$ & 640 & $6.05 \cdot 10^{1}$ \\
$\mathbf{A}_{W, 0}$ & 1 & 6 & $1.46 \cdot 10^{0}$ & 12 & $1.15 \cdot 10^{2}$ \\
& 2 & 31 & $2.67 \cdot 10^{0}$ & 62 & $2.37 \cdot 10^{2}$ \\
& 3 & 141 & $5.45 \cdot 10^{0}$ & 282 & $7.77 \cdot 10^{2}$ \\
$\mathbf{A}_{T, 1}$ & 1 & 25 & $1.36 \cdot 10^{1}$ & 50 & $8.07 \cdot 10^{3}$ \\
& 2 & 110 & $6.80 \cdot 10^{2}$ & 220 & $3.48 \cdot 10^{4}$ \\
& 3 & 460 & $3.04 \cdot 10^{3}$ & 920 & $1.51 \cdot 10^{5}$ \\
\hline
\end{tabular}

Condition Number); the number of Krylov-subspace iterative solver iterations (CG It. or GMRES It.); and the dimensions of the discrete kernels.

Generalized condition numbers were computed as the quotient of largest and smallest non-zero singular values. These quantities are of interest because they are related to the condition numbers one would obtain if computing the Galerkin matrices by discretizing the jump spaces directly. For this reason, we expect that they behave like $\mathscr{O}\left(h^{-1}\right)$ for $\mathbf{A}_{V, \kappa}$ and $\mathbf{A}_{W, \kappa}$, and like $\mathscr{O}\left(h^{-2}\right)$ for $\mathbf{A}_{T, \kappa}$, with mesh width $h \rightarrow 0$. We provide Table 2 at the end of this subsection to illustrate how these quantities behave when the multi-screen is a unit disk, where the jump spaces can be discretized with standard BEM. There we can see that the generalized condition number of the quotient-space BEM matrices has the same growth as the condition number of the standard BEM matrices.

For GMRES/CG, we chose as initial guess $\mathbf{x}_{0}=\mathbf{0}$. As right hand side, we used $\mathbf{r}:=\mathbf{A z}$ with $\mathbf{z}$ a random vector, and $\mathbf{A}$ the Galerkin matrix corresponding to the associated BIE. Singular values, kernel dimensions and all plots were obtained using MATLAB. Moreover, the generalized condition number was computed by regarding every singular value smaller than $10^{-12}$ as zero. For simplicity, and given that the size of the kernels (and thus the feasibility of our approach) does not depend on the wavenumber $\kappa$, we take $\kappa=0$ for the scalar BIEs and $\kappa=1$ for the EFIE. This yields symmetric positive definite Galerkin matrices in the scalar case and allows the use of CG.

Remark 5.1 (Iterative solvers for singular linear systems) We remind that Krylovsubspace iterative solvers can be applied for solving linear systems with singular system matrices as long as they possess a solution, that is, if the right-hand side vector is consistent, see [7,22] and [20, Sect. 6]. 


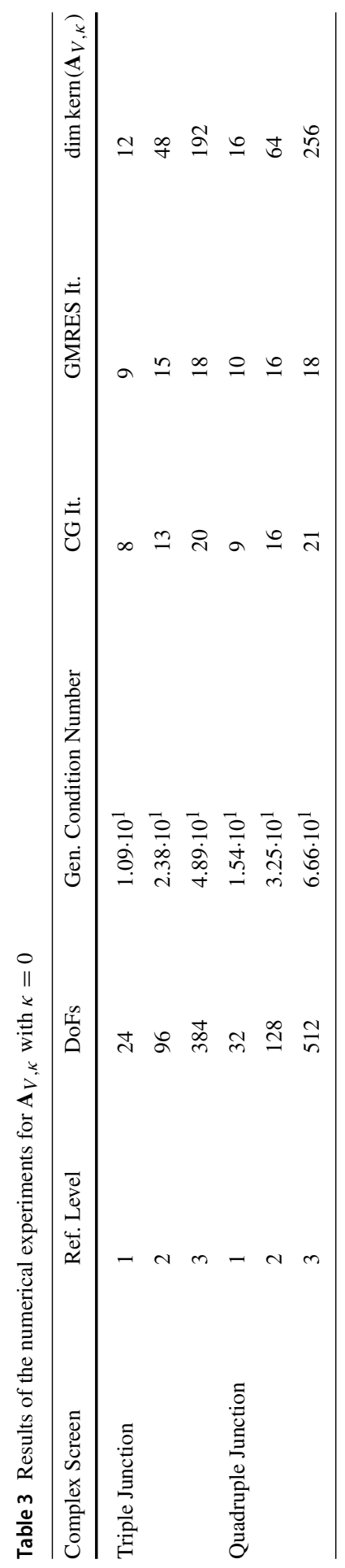




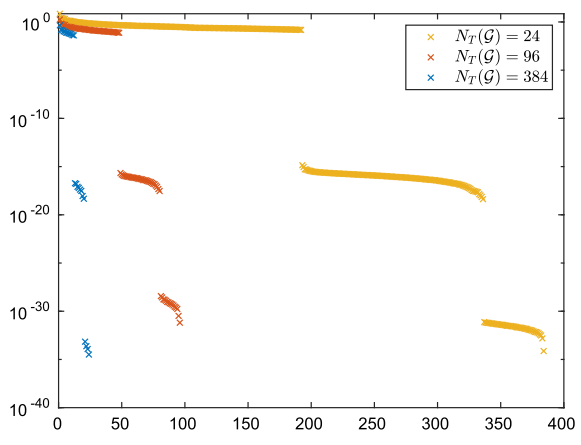

(a) Triple junction

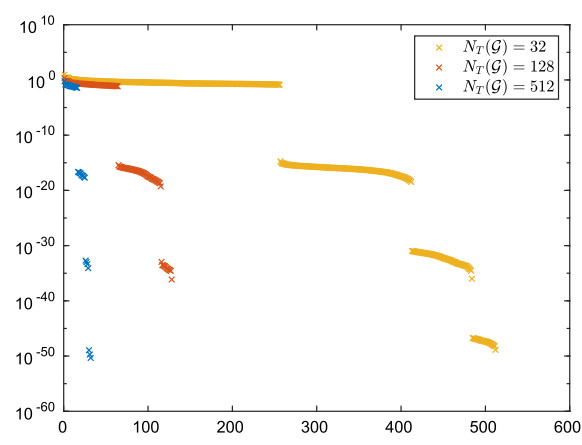

(b) Quadruple junction

Fig. 8 Singular values for the weakly singular operator $\mathbf{A}_{V, \kappa}$ with $\kappa=0$ for different levels of mesh refinement

\subsection{Scalar case: weakly singular and hypersingular BIEs}

First we present the results for the weakly singular BIE. As discussed in Sect. 4.2, we discretize with $\mathscr{S}_{0}^{-1}(\mathscr{T})$. Furthermore, following the discussion in Sect. 4.4, it is clear that dim $\operatorname{kern}\left(\mathbf{A}_{V, \kappa}\right)=N_{V}\left(\mathscr{T}_{0}\right)$, which is exactly what we observe in Table 3. Moreover, Fig. 8 reveals the expected gap between the non-zero singular values of $\mathbf{A}_{V, \kappa}$ corresponding to discrete functions approximating $\widetilde{H}^{-\frac{1}{2}}([\Gamma])$, and the singular values corresponding to the single-trace space $H^{-\frac{1}{2}}([\Gamma])$, which spawn the kernel of $\mathbf{A}_{V, \kappa}$ and are zero up to machine precision.

Table 3 also provides the computed generalized condition number, and the number of CG and GMRES iterations. These quantities are consistent with our expectations, and they confirm that Krylov subspace iterative solvers manage to find a solution to this singular yet consistent linear system.

Next we consider the hypersingular BIE. One may compute the Galerkin matrix $\mathbf{A}_{W, \kappa}$ based on $\mathscr{S}_{1}^{0}(\mathscr{T})$ as described in Sect. 4.2, or, as done in our implementation, one may use $\mathscr{S}_{1,0}^{0}(\mathscr{T}) \subset C^{0}([\Gamma])$ of piecewise linear "continuous" functions on the inflated screen $[\Gamma]$, which are zero at the boundary of $\partial \Gamma$. We remark that this further simplification does not affect the algorithm, as the dropped boundary basis functions belong to $\mathscr{S}_{0}^{-1}(\mathscr{T}) \cap H^{-\frac{1}{2}}([\Gamma])$ and, thus, are contained in $\operatorname{kern}\left(\mathbf{A}_{W, \kappa}\right)$, c.f. Remark 4.3. In other words, they do not affect GMRES. However, due to this choice, the number of degrees of freedom (DoFs) correspond only to the internal vertices of $\mathscr{T}$, and $\operatorname{dim} \operatorname{kern}\left(\mathbf{A}_{W, \kappa}\right)=N_{V}^{*}\left(\mathscr{T}_{0}\right)$, where $N_{V}^{*}\left(\mathscr{T}_{0}\right)$ denotes the number of internal vertices of $\mathscr{T}_{0}$. This is exactly what one sees in Table 4.

Additionally, Fig. 9 shows the singular values of $\mathbf{A}_{W, \kappa}$. There we note the predicted gap between the non-zero singular values (corresponding to discrete functions approximating $\widetilde{H}^{-1 / 2}([\Gamma])$ ), and the singular values that are numerically zero (associated to the single-trace space $\mathscr{S}_{1,0}^{0}\left(\mathscr{T}_{0}\right) \subset H^{-\frac{1}{2}}([\Gamma])$, which is the kernel of $\mathbf{A}_{W, \kappa}$ given our choice of discretization). 


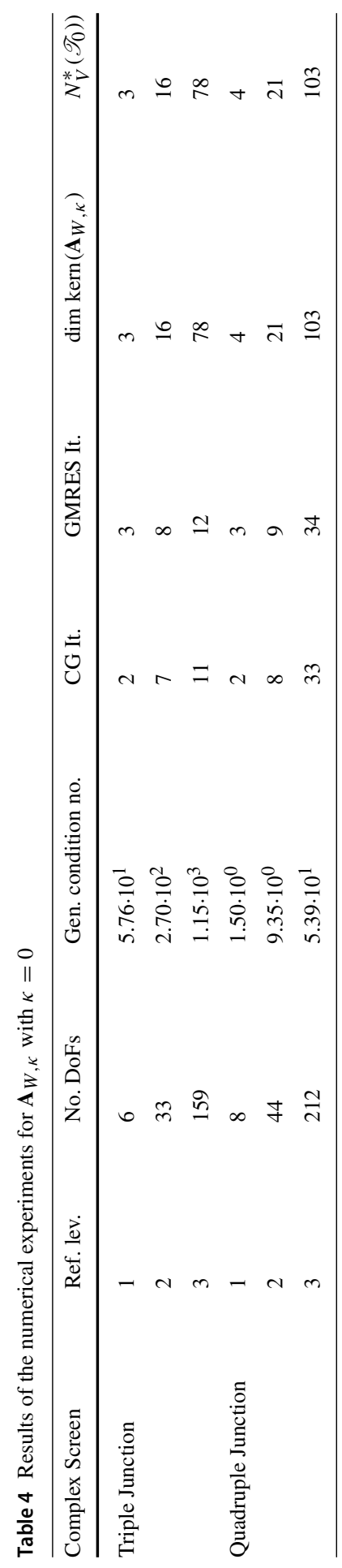




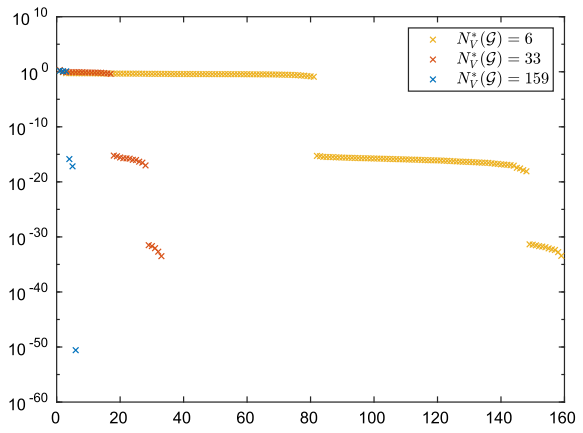

(a) Triple junction

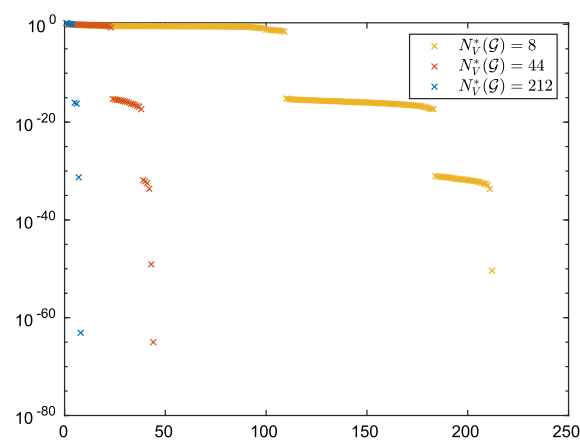

(b) Quadruple junction

Fig. 9 Singular values for the hypersingular operator $\mathbf{A}_{W, \kappa}$ with $\kappa=0$ for different levels of mesh refinement

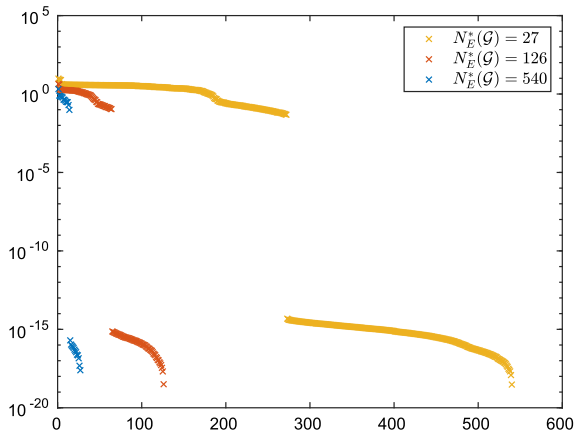

(a) Triple junction

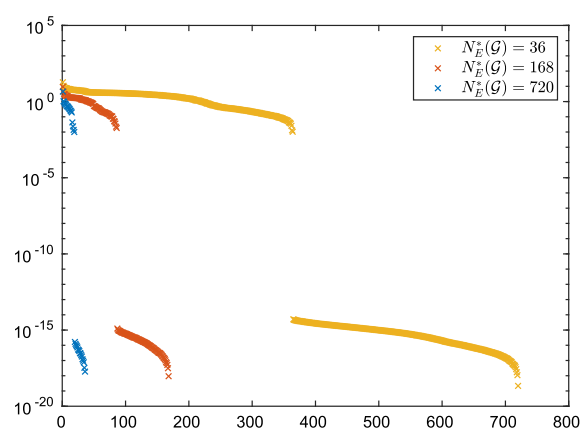

(b) Quadruple junction

Fig. 10 Singular values for the EFIE $\mathbf{A}_{T, \kappa}$ with $\kappa=1$ for different levels of mesh refinement

Table 3 also provides the computed generalized condition number, and the number of CG and GMRES iterations. It is worth noticing that these quantities behave as expected and that both solvers converge.

\subsection{Vectorial case: EFIE}

Finally, we study the EFIE. As for the hypersingular BIE, one can obtain the Galerkin matrix $\mathbf{A}_{T, \kappa}$ relying on the trial/test space $\mathscr{N}_{0}(\mathscr{T})$ as in Sect. 4.2, or one may use the space $\mathscr{N}_{0,0}(\mathscr{T}) \subset \mathbb{H}^{-\frac{1}{2}}\left(\operatorname{curl}_{\Gamma}, \Gamma\right)$ of edge element functions on the inflated screen $[\Gamma]$, which vanish at the boundary of $\partial[\Gamma]$. As before, the algorithm is not affected by this further simplification, since the dropped boundary basis functions belong to $\mathscr{N}_{0}(\mathscr{T}) \cap \mathbf{H}^{-\frac{1}{2}}\left(\operatorname{curl}_{\Gamma},[\Gamma]\right)$ and, hence, just contribute to $\operatorname{kern}\left(\mathbf{A}_{T, \kappa}\right)$. In this approach the number of degrees of freedom (DoFs) in Table 3 agrees with that of the internal edges of $\mathscr{T}$. Consequently, $\operatorname{dim} \operatorname{kern}\left(\mathbf{A}_{T}\right)=N_{E}^{*}\left(\mathscr{T}_{0}\right)$, with $N_{E}^{*}\left(\mathscr{T}_{0}\right)$ the number of internal edges of $\mathscr{T}_{0}$. This is verified by our numerical experiments. 


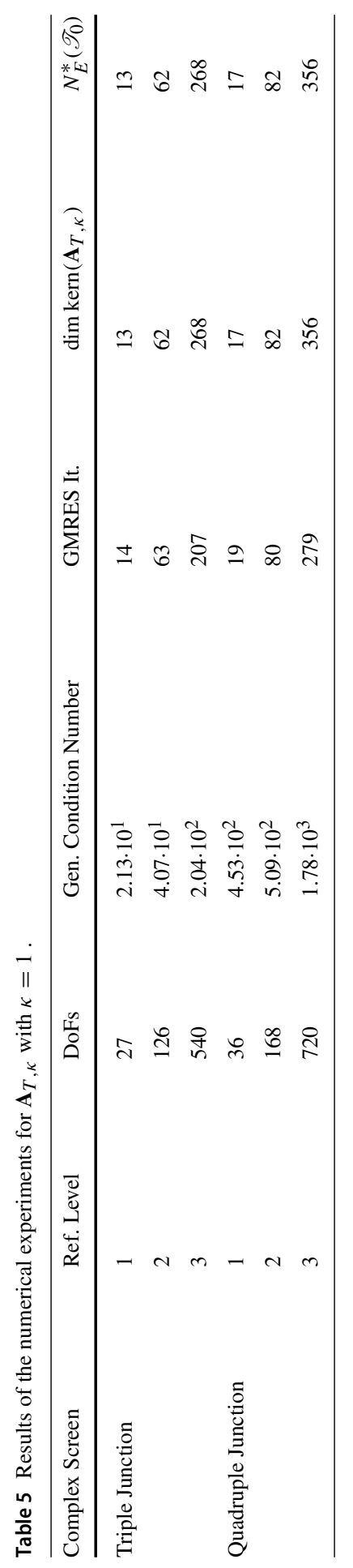


We plot the singular values of $\mathbf{A}_{T, \kappa}$ in Fig. 10. As before, we find a clear distinction between the singular values belonging to the kernel and those corresponding to the jump space. Similarly, Table 5 reveals that GMRES works as predicted for this setting, too.

Funding Open access funding provided by Swiss Federal Institute of Technology Zurich.

Open Access This article is licensed under a Creative Commons Attribution 4.0 International License, which permits use, sharing, adaptation, distribution and reproduction in any medium or format, as long as you give appropriate credit to the original author(s) and the source, provide a link to the Creative Commons licence, and indicate if changes were made. The images or other third party material in this article are included in the article's Creative Commons licence, unless indicated otherwise in a credit line to the material. If material is not included in the article's Creative Commons licence and your intended use is not permitted by statutory regulation or exceeds the permitted use, you will need to obtain permission directly from the copyright holder. To view a copy of this licence, visit http://creativecommons.org/licenses/by/4.0/.

\section{References}

1. Babuška, I., Guo, B.Q., Stephan, E.P.: On the exponential convergence of the $h$ - $p$ version for boundary element Galerkin methods on polygons. Math. Methods Appl. Sci. 12(5), 413-427 (1990). https://doi. org/10.1002/mma.1670120506

2. Bespalov, A., Heuer, N., Hiptmair, R.: Convergence of natural $h p$-BEM for the electric field integral equation on polyhedral surfaces. SIAM J. Numer. Anal. 48(4), 1518-1529 (2010). https://doi.org/10. $1137 / 090766620$

3. Buffa, A.: Remarks on the discretization of some non-positive operators with application to heterogeneous Maxwell problems. SIAM J. Numer. Anal. 43(1), 1-18 (2005)

4. Buffa, A., Christiansen, S.H.: The electric field integral equation on Lipschitz screens: definitions and numerical approximation. Numer. Math. 94(2), 229-267 (2003). https://doi.org/10.1007/s00211-0020422-0

5. Buffa, A., Hiptmair, R.: Galerkin boundary element methods for electromagnetic scattering. In: Topics in Computational Wave Propagation. Lecture Notes in Computational Science and Engineering, vol. 31, pp. 83-124. Springer, Berlin (2003). https://doi.org/10.1007/978-3-642-55483-4_3

6. Carr, M., Topsakal, E., Volakis, J.: A procedure for modeling material junctions in 3-d surface integral equation approaches. IEEE Trans. Antennas Propag. 52(5), 1374-1378 (2004). https://doi.org/10.1109/ TAP.2004.827247

7. Choi, S.C.T.: Iterative methods for singular linear equations and least-squares problems. ProQuest LLC, Ann Arbor, MI (2007). Thesis (Ph.D.), Stanford University

8. Claeys, X., Hiptmair, R.: Integral equations on multi-screens. Integr. Equ. Oper. Theory 77(2), 167-197 (2013). https://doi.org/10.1007/s00020-013-2085-X

9. Claeys, X., Hiptmair, R.: Integral equations for electromagnetic scattering at multi-screens. Integr. Equ. Oper. Theory 84(1), 33-68 (2016). https://doi.org/10.1007/s00020-015-2242-5

10. Cools, K.: Mortar boundary elements for the EFIE applied to the analysis of scattering by PEC junctions. In: 2012 Asia-Pacific Symposium on Electromagnetic Compatibility (APEMC), pp. 165-168 (2012). https://doi.org/10.1109/APEMC.2012.6237847

11. Costabel, M., Dauge, M.: General edge asymptotics of solutions of second-order elliptic boundary value problems I, II. Proc. R. Soc. Edinb. Sect. A 123(1), 109-155, 157-184 (1993). https://doi.org/ $10.1017 / \mathrm{S} 0308210500021272$

12. Costabel, M., Dauge, M., Duduchava, R.: Asymptotics without logarithmic terms for crack problems. Commun. Partial Differ. Equ. 28(5-6), 869-926 (2003). https://doi.org/10.1081/PDE-120021180

13. Dauge, M.: Elliptic Boundary Value Problems on Corner Domains. Lecture Notes in Mathematics, vol. 1341. Springer, Berlin (1988)

14. Ervin, V.J., Stephan, E.P.: A boundary element Galerkin method for a hypersingular integral equation on open surfaces. Math. Methods Appl. Sci. 13(4), 281-289 (1990). https://doi.org/10.1002/mma. 1670130402 
15. Ervin, V.J., Stephan, E.P., El-Seoud, S.A.: An improved boundary element method for the charge density of a thin electrified plate in $\mathbb{R}^{3}$. Math. Methods Appl. Sci. 13(4), 291-303 (1990). https://doi. org/10.1002/mma.1670130403

16. Gimperlein, H., Meyer, F., Özdemir, C., Stark, D., Stephan, E.P.: Boundary elements with mesh refinements for the wave equation. Numer. Math. 139(4), 867-912 (2018). https://doi.org/10.1007/s00211018-0954-6

17. Girault, V., Raviart, P.: Finite Element Methods for Navier-Stokes Equations. Springer, Berlin (1986)

18. Gómez-Sousa, H., nos López, O.R., Martínez-Lorenzo, J.: Junction modeling for piecewise nonhomogeneous geometries involving arbitrary materials. In: 2014 IEEE Antennas and Propagation Society International Symposium (APSURSI), pp. 2196-2197 (2014). https://doi.org/10.1109/APS. 2014.6905425

19. Gwinner, J., Stephan, E.P.: Advanced Boundary Element Methods. Springer Series in Computational Mathematics, vol. 52. Springer, Cham (2018). https://doi.org/10.1007/978-3-319-92001-6

20. Hanke, M.: Conjugate Gradient Type Methods for Ill-Posed Problems. Pitman Research Notes in Mathematics Series, vol. 327. Longman Scientific \& Technical, Harlow (1995)

21. Hiptmair, R., Kielhorn, L.: Betl: a generic boundary element template library. Technical Report 201236, Seminar for Applied Mathematics, ETH Zürich, Switzerland (2012). https://www.sam.math.ethz. ch/sam_reports/reports_final/reports2012/2012-36.pdf

22. Hochbruck, M., Lubich, C.: Error analysis of Krylov methods in a nutshell. SIAM J. Sci. Comput. 19, 695-701 (1998)

23. Maz'ya, V., Rossmann, J.: Elliptic equations in polyhedral domains. In: Mathematical Surveys and Monographs, vol. 162. American Mathematical Society, Providence, RI (2010). https://doi.org/10. 1090/surv/162

24. McLean, W.: Strongly Elliptic Systems and Boundary Integral Equations. Cambridge University Press, Cambridge (2000)

25. Nazarov, S.A., Plamenevsky, B.A.: Elliptic problems in domains with piecewise smooth boundaries, De Gruyter Expositions in Mathematics, vol. 13. Walter de Gruyter \& Co., Berlin (1994). https://doi. org/10.1515/9783110848915.525

26. von Petersdorff, T.: Randwertprobleme der elastizitätstheorie für polyeder - singularitäten und approximation mit randelementmethoden. Ph.D. thesis, Tu Darmstadt (1989)

27. von Petersdorff, T., Stephan, E.P.: Decompositions in edge and corner singularities for the solution of the Dirichlet problem of the Laplacian in a polyhedron. Math. Nachr. 149, 71-103 (1990). https://doi. org/10.1002/mana.19901490106

28. von Petersdorff, T., Stephan, E.P.: Regularity of mixed boundary value problems in $R^{3}$ and boundary element methods on graded meshes. Math. Methods Appl. Sci. 12(3), 229-249 (1990). https://doi.org/ 10.1002/mma.1670120306

29. von Petersdorff, T., Stephan, E.P.: Singularities of the solution of the Laplacian in domains with circular edges. Appl. Anal. 45(1-4), 281-294 (1992). https://doi.org/10.1080/00036819208840102

30. Putnam, J., Medgyesi-Mitschang, L.: Combined field integral equation formulation for inhomogeneous two and three-dimensional bodies: the junction problem. IEEE Trans. Antennas Propag. 39(5), 667-672 (1991). https://doi.org/10.1109/8.81498

31. Sauter, S.A., Schwab, C.: Boundary element methods, Springer Series in Computational Mathematics, vol. 39. Springer, Berlin (2011). https://doi.org/10.1007/978-3-540-68093-2

32. Schwab, C., Suri, M.: The optimal $p$-version approximation of singularities on polyhedra in the boundary element method. SIAM J. Numer. Anal. 33(2), 729-759 (1996). https://doi.org/10.1137/0733037

33. Stephan, E.: Boundary integral equations for screen problems in $\mathbb{R}^{3}$. Integr. Equ. Oper. Theory $\mathbf{1 0}(2)$, 236-257 (1987)

34. Urzúa-Torres, C.: Operator preconditioning for galerking boundary element methods on screens. Eth dissertation, ETH Zurich, Switzerland (2018)

35. Ylä-Oijala, P., Taskinen, M., Sarvas, J.: Surface integral equation method for general composite metallic and dielectric structures with junctions. PIER 52, 81-108 (2005). https://doi.org/10.2528/ PIER04071301

Publisher's Note Springer Nature remains neutral with regard to jurisdictional claims in published maps and institutional affiliations. 